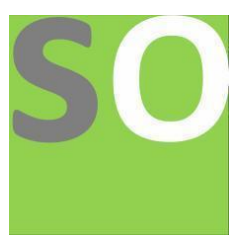

Article title: Relativistic Equation Failure for LIGO Signals

Authors: XD Dongfang[1]

Affiliations: Wutong Mountain National Forest Park[1]

Orcid ids: 0000-0002-3644-5170[1]

Contact e-mail: xddongfang@gmail.com

License information: This work has been published open access under Creative Commons Attribution License http://creativecommons.org/licenses/by/4.0/, which permits unrestricted use, distribution, and reproduction in any medium, provided the original work is properly cited. Conditions, terms of use and publishing policy can be found at https://www.scienceopen.com/.

Preprint statement: This article is a preprint and has not been peer-reviewed, under consideration and submitted to ScienceOpen Preprints for open peer review.

DOI: 10.14293/S2199-1006.1.SOR-.PPBWE7H.v1

Preprint first posted online: 14 January 2021

Keywords: GW150914, Lagrange frequency change rate, Blanchet frequency equation, com quantization, LIGO 


\title{
Relativistic Equation Failure for LIGO Signals
}

\author{
X. D. Dongfang \\ Wutong Mountain National Forest Park, Shenzhen, China
}

\begin{abstract}
Signal waves of the monotone increasing frequency detected by LIGO are universally considered to be gravitational waves of spiral binary stars, and the general theory of relativity is thus universally considered to have been confirmed by the experiments. Here we present a universal method for signal wave spectrum analysis, introducing the true conclusions of numerical calculation and image analysis of GW150914 signal wave. Firstly, numerical calculation results of GW150914 signal wave frequency change rate obey the com quantization law which needs to be accurately described by integers, and there is an irreconcilable difference between the results and the generalized relativistic frequency equation of the gravitational wave. Secondly, the assignment of the frequency and frequency change rate of GW10914 signal wave to the generalized relativistic frequency equation of gravitational wave constructs a non-linear equation group about the mass of wave source, and the computer image solution shows that the equation group has no GW10914 signal wave solution. Thirdly, it is not unique to calculate the chirp mass of the wave source from the different frequencies and change rates of the numerical relativistic waveform of the GW150914 signal wave, and the numerical relativistic waveform of the GW150914 signal wave deviates too far from the original waveform actually. Other LIGO signal waveforms do not have obvious characteristics of gravitational frequency variation of spiral binary stars and lack precise data, so they cannot be used for numerical analysis and image solution. Therefore, LIGO signals represented by gw50914 signal do not support the relativistic gravitational wave frequency equation. However, whether gravitational wave signals from spiral binaries that may be detected in the future follow the same co quantization law? The answer is not clear at present.
\end{abstract}

Keywords: GW150914 signal wave; Lagrange frequency change rate; Blanchet frequency equation; com quantization.

\section{Introduction}

In 1915, Einstein established the equation of a gravitational field ${ }^{[1]}$ and founded general relativity ${ }^{[2]}$. In 1916, Einstein predicted the existence of gravitational waves based on general theory of relativity, and published the first paper on gravitational waves ${ }^{[3]}$. According to the general theory of relativity, when an object accelerates, it will generate gravitational radiation and escape from the gravitational field source and propagate in the vacuum to form gravitational waves, which is considered that accelerated masses stimulate fluctuations in space-time. In fact, gravitational waves are fluctuating gravitational fields. In 1916, Schwarzschild calculated the static spherical symmetric solution ${ }^{[4,5]}$ of the Einstein field equation, and the singularity in this particular solution was interpreted as the radius of the black hole's horizon. In February 1918, Einstein published the second paper on gravitational waves and gave formulas for the calculation of gravitational radiant energy ${ }^{6]}$. In 1963, Kerr found the solution of the rotating black hole ${ }^{[7]}$ for the field equation.

Since Einstein proposed the concept of gravitational waves, the theoretical and experimental detection principles of gravitational waves ${ }^{[8-10]}$ have been continuously developed within the framework of general relativity. Although gravitational waves exist widely, they are generally difficult to detect due to their very weak energy. According to theoretical predictions, massive binary black holes or binary compact stars can generate powerful energy gravitational waves when merger, so compact binary stars like binary black holes become an important model for the study of gravitational wave theory and experimental detection principles. The main vibration frequency and energy of the gravitational waves generated by the binary star during the process of inspiral, merger and ringdown first increase and then decrease with time ${ }^{[11,12]}$. In 1974, Hulse and Taylor used a radio telescope to find a pulsed binary neutron star that rotates one revolution every 8 hours $^{[13]}$. The radiation of gravitational waves in the process of binary satellite spiralling reduces the system energy and decreases the revolution period. Precise measurement results show that the period of revolution of the twin neutron star is reduced by $10^{-4}$ second per year, which is in accordance with the theoretical value. The discovery of the pulsed binary neutron star is considered to prove indirectly the existence of the gravitational wave. In 1995, Blanchet derived the Blanchet frequency equation ${ }^{[14]}$ of gravitational wave from the binary star wave source, which is one of the important corollaries of the general relativistic gravitational wave theory.

In February 2016, LIGO's two detectors at Livingston and Hanford received the signal wave named GW150914 $4^{[15]}$ at $6.9 \mathrm{~ms}$ intervals on September 14, 2015. In the signal waveforms, the vibration curve with the strain not exceeding about $1.2 \times 10^{-21}$ for a period of time from $0.25 \mathrm{~s}$ to 0.45 $\mathrm{s}$ is considered as the gravitational wave of the spiral binary black holes, which was generated by the merger of two black holes whose mass was $29_{-4}^{+4} M_{\odot}$ and $36_{-4}^{+5} M_{\odot}$ respectively from the solar system 1.5 billion light years away, 1.3 billion years ago, and the mass of the merged black hole is $62_{-4}^{+4} M_{\odot}^{[16]}$. In less than two years after that, LIGO 
successively announced the detection of four gravitational wave signals GW151226 $6^{[17]}$, GW170104 ${ }^{[18]}, \mathrm{GW} 170814^{[19]}$, and GW170817 $7^{[20]}$ from the spiral binary holes or spiral binary neutron stars. The widely accepted conclusion is that these signals are gravitational waves generated by the merging of spiral binary black holes or spiral binary neutron stars. They constitute the last piece of Einstein's general relativity. Not only does the black hole predicted by general relativity exist, but the binary black holes also merge frequently, even though space- time is ancientfar. Why do all the gravitational wave signals detect by LIGO come from the merger of ancient-far binary black holes or binary neutron stars? How is the detailed frequency law of the gravitational wave from binary stars? Scientific assertions require scientific argumentation. The vibration curve of signal wave of GW150914 is clear, and the part where the frequency changes monotonically accords with the characteristics of spiral binaries. However, the frequency distribution of signals such as GW151226, GW170104, GW170814, GW170817 does not have precise rules, and quantitative calculations cannot be made to arrive at reliable conclusions, and there is actually a lot of uncertainty. Therefore, up to now, only gw150914 signal wave can be used to quantitatively explain the degree of LIGO signal conforming to the generalized relativistic gravitational wave equation.

Here, data of gw150914 signal wave is analyzed in detail. First, the time of main strain peak is extracted from gw150914 signal wave database, and the time of wide peak and uncertain peak is corrected within the error range, and the frequency of main strain peak is determined. Then, the Lagrange frequency change rate, which represents the average frequency change rate, is calculated. It is proved that the frequency change of GW1500914 signal wave presents a generalized quantization law called com quantum law which needs to be described by integers, but it does not support the generalized relativistic Blanchet frequency equation of a gravitational wave. Thirdly, the broken line of frequency average change rate of GW150914 signal wave is compared with the function curve of Blanchet frequency equation by using the image method. It is further proved that the generalized relativistic Blanchet frequency equation of GW150914 signal wave, which is regarded as gravitational wave of spiral binary stars, is invalid. Finally, the frequency change rate of LIGO's numerical relativistic gravitational waveform is calculated, and the result also does not conform to the general relativistic Blanchet frequency equation.

\section{Frequency distribution and change laws of GW150914 signal wave}

The frequency of the main vibration part of the GW150914 signal wave increases monotonically, and the strain of the main vibration part shows a tendency to change synchronously with the frequency, but does not increase strict- ly monotonously, which is mainly caused by noise. The detection of gravitational waves usually uses filtering techniques to shield the noise. It should be pointed out that the filtering technology can only shield the noise of the expected frequency distribution, and the isolated wave or the noise of unintended frequency distribution can stil1 reach the detector. The noise with similar energy mixed in the gravitational wave strengthens or weakens the strain at a certain moment, so that the gravitational waveform is distorted to varying degrees. The gravitational wave waveform is also distorted by the forced vibration of the detection instrument due to the tremor of the crust without a fixed frequency. Therefore, the strain corresponding to the gravitational wave signal may be shifted or masked. Strain distribution of the GW150914 signal wave is more complex than its frequency distribution. Here we mainly analyze its frequency distribution and its variation laws.

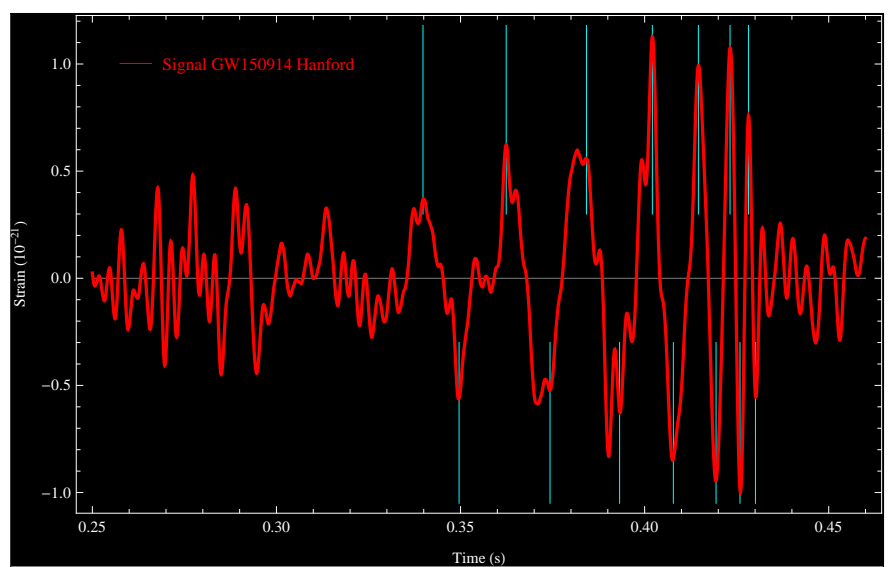

Figure 1 The positive and negative strain time of the GW150914 signal wave ${ }^{[21]}$.

As shown in Figure 1, the main positive and negative strain peak time are extracted from the Hanford database $^{[21]}$ of the GW150914 signal wave and marked by vertical lines in reverse time sequence. The rightmost vertical line corresponds to the sequence number 1 . Correct the time of wide or uncertain peak within the allowable range of error. The accuracy of recording time in LIGO database reaches $10^{-9} \mathrm{~s}$. The correction time of wide or uncertain peaks are obtained by using the characteristic equation correction method and the accuracy of $10^{-9} \mathrm{~s}$ is naturally retained. The formulas for the period and frequency are $T_{i}=t_{i}-t_{i+1}$ and $f_{i}=1 / T_{i}$, respectively. The time of the positive and negative strains, the corresponding period, and the frequency calculation result are listed in Table 1 in reversed time order. The positive strain peaks have 6 main frequencies from $36.55320819 \mathrm{~Hz}$ to $197.3975904 \mathrm{~Hz}$; the negative strain peaks have a total of seven main frequencies from $35.37953557 \mathrm{~Hz}$ to $230.7600891 \mathrm{~Hz}$. The period and frequency of the strain peaks characterize the period and frequency of the signal wave.

As shown in Figure 2, according to the positive and 
negative strain time $t_{i}$ and frequency $f_{i}$ listed in Table 1 , the frequency and time curves of the positive and negative strains of the GW150914 signal wave are plotted respectively. The two polylines have the same monotonous change trend, and they are very close to each other. A single polyline that plots the frequency and time of the positive and negative strain mixtures reflects the fluctuation characteristics of the frequency distribution.

Table 1 Positive and negative strain peak times of the GW150914 signal wave and its frequency distribution

\begin{tabular}{c|ccc|ccc}
\hline \multirow{2}{*}{$i$} & \multicolumn{2}{|c|}{ Positive strain observation values } & \multicolumn{2}{c}{ Negative strain observation values } \\
\cline { 2 - 6 } & $t_{i} / \mathrm{s}$ & $T_{i} / \mathrm{s}$ & $f_{i} / \mathrm{Hz}$ & $t_{i} / \mathrm{s}$ & $T_{i} / \mathrm{s}$ & $f_{i} / \mathrm{Hz}$ \\
\hline 1 & 0.428222656 & 0.005065918 & 197.3975904 & 0.430297903 & 0.004333505 & 230.7600891 \\
2 & 0.423156738 & 0.008573092 & 116.6440307 & 0.425964398 & 0.007333624 & 136.3582345 \\
3 & 0.414583646 & 0.012607488 & 79.31794085 & 0.418630774 & 0.010784741 & 92.72359945 \\
4 & 0.401976158 & 0.017110162 & 58.44479852 & 0.407846033 & 0.014636434 & 68.32265222 \\
5 & 0.384865996 & 0.022037889 & 45.37639637 & 0.393209599 & 0.018851727 & 53.04553744 \\
6 & 0.362828106 & 0.027357380 & 36.55320819 & 0.374357872 & 0.023402144 & 42.73112738 \\
7 & 0.335470727 & & 0.350955728 & 0.028264927 & 35.37953557 \\
8 & & & 0.322690801 & \\
\hline
\end{tabular}

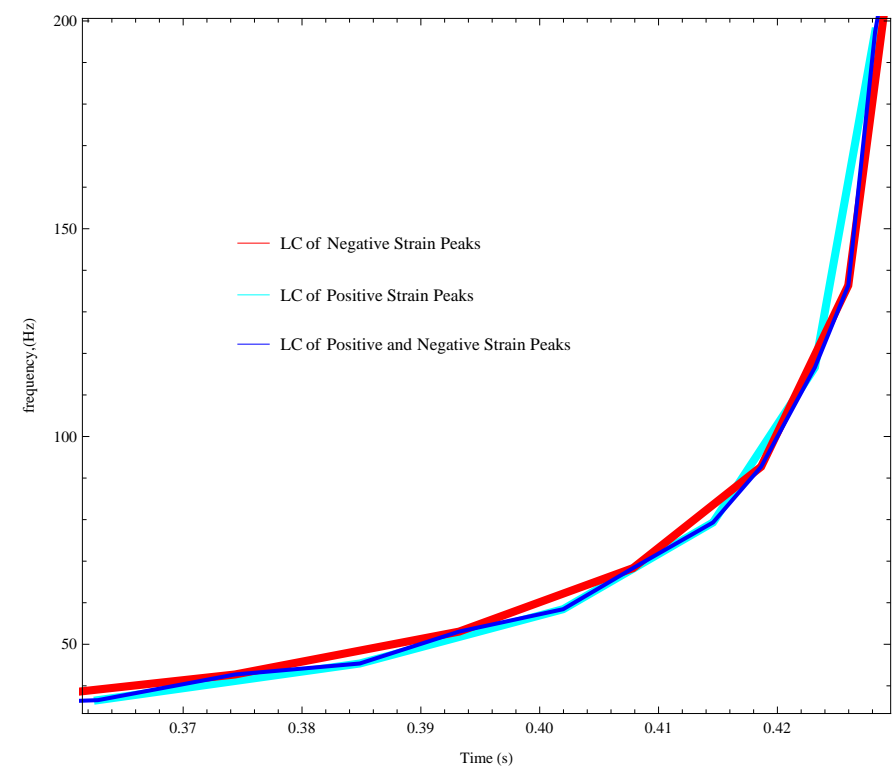

Figure 2 Frequency-time polylines of strain peaks of the GW150914 signal wave.

It is assumed that the signal wave of GW150914 is the gravitational wave of a helical double black hole. In order to determine the chirp mass of GW150914 wave source, LIGO uses the low frequency approximation of the highly nonlinear Blanchet frequency equation of general relativity. In fact, the high and low frequencies are relative and there is no clear demarcation. To judge whether the frequency distribution and variation laws of a signal wave accord with the Blanchet frequency equation, it is necessary to calculate the time derivative of the frequency. The frequencies of the GW150914 signal wave and their change rates are all discrete. The classical method of calculation is to first modify the graph of frequency change over time into a smooth curve, draw the tangent of the position of each frequency, and then measure the slope of these tangents. Therefore, the derivative of the frequency versus time $\dot{f}_{i}$ at each frequency is obtained. However, the correction of a polyline to a smooth curve has great uncertainty, and the tangent line also has uncertainty. The calculation of the change rate is actually uncertain. As we all know, the mathematical significance of the average rate of change of the discrete variation is consistent with the Lagrange mean value theorem ${ }^{[22]}$, so the Lagrange mean value theorem can be used to calculate the time derivative of the frequency of the gravitational wave, that is, the Lagrange frequency change rate. The integer $i$ in a table is an inverse time sequence, and the variation rate of discrete frequency conforming to the meaning of Lagrange mean value theorem is called Lagrange frequency change rate. It is defined as,

$$
\dot{f}_{i}=\frac{f_{i-1}-f_{i+1}}{T_{i}+T_{i-1}}
$$

The Lagrange change rate has a definite value for calculating the average change rate of the discrete frequency, which is better than the tangent slope measured after the frequency time polyline is corrected to a smooth curve, because the correction of the curve and the measurement results of the slope are uncertain.

The column in Table 2 that $\dot{f}_{i}$ is located lists the Lagrange frequency change rates of the main positive and negative strain peaks of the GW150914 signal wave. According to dimension analysis, the time derivative of the frequency should be represented by the square of the frequency. The column in Table 2 where $\dot{f}_{i} \dot{f}_{i}^{-2}$ is located also lists the ratio of the frequency change rate of the positive and negative strains of the GW150914 signal wave to their square of the frequency. Numerical results show that the frequency distribution and changes in the positive and negative strain peaks are different, but the ratio $\dot{f}_{i} \dot{f}_{i}^{-2}$ has the same distribution with high accuracy, which 
is expressed as the equation of following form,

$$
\left\{\begin{array}{c}
\dot{f}_{2}^{+}=0.636307692 f_{2}^{2} \\
\dot{f}_{3}^{+}=0.436753649 f_{3}^{2} \\
\dot{f}_{4}^{+}=0.334368530 f_{4}^{2} \\
\dot{f}_{5}^{+}=0.271585859 f_{5}^{2} \\
\vdots
\end{array}\right\} \Leftrightarrow\left\{\begin{array}{c}
\dot{f}_{2}^{-}=0.636307692 f_{2}^{2} \\
\dot{f}_{3}^{-}=0.436753649 f_{3}^{2} \\
\dot{f}_{4}^{-}=0.334368530 f_{4}^{2} \\
\dot{f}_{5}^{-}=0.271585859 f_{5}^{2} \\
\dot{f}_{6}^{-}=0.228972362 f_{6}^{2}
\end{array}\right\}
$$

The positive and negative superscripts represent the positive and negative strains, respectively, respectively. Dis- crete laws of physical quantities need to be described by integers. Discrete laws are essentially generalized quantization laws including quantization laws, which are called com quantum laws. Equation (2) shows that the discrete frequencies of the GW150914 signal wave imply a generalized quantization law closely related to quantum numbers in accordance with the dimensional law. This opens the prelude to the gravitational com quantum theory, which systematically describes the quantization law of gravitational systems. It also means that the GW150914 gravitational wave does not correspond to the Blanchet frequency equation of general relativity.

Table 2 Relativistic differences of frequency change rule of the GW150914 signal wave

\begin{tabular}{|c|c|c|c|c|c|c|c|c|}
\hline \multirow{2}{*}{$i$} & \multicolumn{4}{|c|}{ Positive strain observation values } & \multicolumn{4}{|c|}{ Negative strain observation values } \\
\hline & $f_{i}$ & $\dot{f}_{i}$ & $\dot{f}_{i} / f_{i}^{2}$ & $\dot{f}_{i} / f_{i}^{11 / 3}$ & $f_{i}$ & $\dot{f}_{i}$ & $\dot{f}_{i} / f_{i}^{2}$ & $\dot{f}_{i} / f_{i}^{11 / 3}$ \\
\hline 1 & 197.3975904 & & & & 230.7600891 & & & \\
\hline 2 & 116.6440307 & 8657.49422 & 0.636307692 & 0.000228505 & 136.3582345 & 11831.23042 & 0.636307692 & 0.000176143 \\
\hline 3 & 79.31794085 & 2747.763841 & 0.436753649 & 0.000298275 & 92.72359945 & 3755.061 & 0.436753649 & 0.000229925 \\
\hline 4 & 58.44479852 & 1142.134177 & 0.33436853 & 0.000379882 & 68.32265222 & 1560.827218 & 0.33436853 & 0.000292831 \\
\hline 5 & 45.37639637 & 559.1999942 & 0.271585859 & 0.000470461 & 53.04553744 & 764.1961764 & 0.271585859 & 0.000362654 \\
\hline 6 & 36.55320819 & & & & 42.73112738 & 418.0919129 & 0.228972362 & 0.000438405 \\
\hline 7 & & & & & 35.37953557 & & & \\
\hline
\end{tabular}

\section{Numerical Proof of Relativistic Equation Failure for GW150914 Signal wave}

According to the theory of general relativity, frequency distribution and variation law of a gravitational wave of spiral binaries are highly nonlinear Blanchet frequency equations ${ }^{[14]}$, which can not be solved accurately. The frequency and strain of the signal wave increase monotonously, which is only qualitatively characterized by the merging of spiral binaries. GW150914 signal was regarded as gravitational wave of spiral double black hole only because of its qualitative characteristics. In the literature, the zero order approximate equation of relativistic Blanchet frequency equation ${ }^{[15]}$ at low frequency

$$
\dot{f}=\frac{96 \pi^{8 / 3} G^{5 / 3} m_{1} m_{2}}{5 c^{5}\left(m_{1}+m_{2}\right)^{1 / 3}} f^{11 / 3}
$$

is used to infer the mass of binary black holes before merging. Among them, the universal gravitational constant is $G=6.674 \times 10^{-11} \mathrm{~m}^{3} \mathrm{~kg}^{-1} \mathrm{~s}^{-2}$, the speed of light in a vacuum is $c=2.998 \times 10^{8} \mathrm{~m} \mathrm{~s}^{-1}$, and $m_{1}$ and $m_{1}$ is the masses of two black holes in binary black hole's gravitational wave source respectively. However, low frequency approximation is not a scientific method. Because lowfrequency and high-frequency are only relative, there is no clear boundary between them. Theoretically, the frequency conversion motion has a low frequency approaching to zero. Low frequency approximation (3) makes it easy for readers to shift their attention to the so-called chirp mass, while ignoring the difference between the frequency distribution of the GW150914 signal wave and the Blanchet frequency equation.
Note that the approximate theoretical value $\dot{f} \propto f^{11 / 3}$ derived from the formula (3) does not conform to the observed value (2) clearly. The low-frequency approximation of the Blanchet frequency equation requires $\dot{f}_{i} \dot{f}_{i}^{-11 / 3}$ to be an approximate constant, but the $\dot{f}_{i} \dot{f}_{i}^{-11 / 3}$ value of the GW150914 signal wave listed in Table 2 varies with frequency, which is a prominent contradiction, manifesting as that the first significant digits of the $\dot{f}_{i} \dot{f}_{i}^{-11 / 3}$ values corresponding to each frequency of positive and negative strain peaks are quite different. This difference cannot be eliminated by a numerical method that corrects the strain peak time or redefines the change rate of the discrete frequency. Although Equation (3) is the zero-order approximation of the Blanchet's frequency equation at low frequencies, the zero-order approximation embodies the main rule of the Blanchet's frequency equation. The high-order approximation of the Blanchet frequency equation has a small effect on the first significant figure, and it cannot change the conclusion that the $\dot{f}_{i} \dot{f}_{i}^{-11 / 3}$ values of the GW150914 signal wave are not constant and it are inconsistent with the Blanchet equation.

The amplitudes and orbital contraction rates of the general relativistic quadrupole moments of binary stars with unequal masses are related to the system's mass, which happens to be in the zero-order approximation (3) of the Blanchet's frequency equation,

$$
\mathscr{M}=\frac{\left(m_{1} m_{2}\right)^{3 / 5}}{\left(m_{1}+m_{2}\right)^{1 / 5}}=\frac{c^{3}}{G}\left(\frac{5}{96} \pi^{-8 / 3} f^{-11 / 3} \dot{f}\right)^{3 / 5}
$$

According to the report of LIGO, the masses of the two black holes of the GW150914 signal source are $29_{-4}^{+4} M_{\odot}$ and $36_{-4}^{+5} M_{\odot}$, respectively, where $M_{\odot}$ represents the mass 
of the sun. From this, the estimated value of the chirp mass of the wave is

$$
\mathscr{M}_{\text {LIGO }}=28.10_{-3.51}^{+3.89} M_{\odot}
$$

If the GW150914 signal wave is the gravitational wave radiated by a pair of spiral binary stars, the results of chirp mass calculated by different frequencies of the gravitational waves should be approximately equal, because the chirp mass of a spiral binary star is unique. However, according to the lower frequency $f_{5}=45.37639637 \mathrm{~Hz}$ of the positive strain of the GW150914 signal wave and its Lagrange change rate $\dot{f}_{5}=559.1999942 \mathrm{Hzs}^{-1}$ listed in Table 2, it is estimated that the chirp mass of the signal wave source is $\mathscr{M}=36.090 M_{\odot}$, and according to the low- er frequency $f_{6}=42.73112738 \mathrm{~Hz}$ of the negative strain of the GW150914 signal wave and its Lagrange change rate $\dot{f}_{6}=418.0919129 \mathrm{Hzs}^{-1}$, it is estimated that the chirp mass of the signal wave source is $\mathscr{M}=30.873 M_{\odot}$. It can be seen that the estimation of chirp masses from differen$\mathrm{t}$ frequencies are very different, and there is no basis for making trade-offs. This poses a challenge to the general relativity Blanchet frequency equation. There are still unrecorded lower frequencies of the signal wave, so there must be other different estimates of the mass of the defect. Using the frequencies of all main positive and negative strain peaks of the GW150914 signal wave and their time derivatives, the estimated chirp masses of the wave source are respectively as follows,

$$
\begin{aligned}
\mathscr{M}_{2}^{+}= & \frac{\left(2.998 \times 10^{8}\right)^{3}}{6.674 \times 10^{-11}}\left(\frac{5}{96} \pi^{-8 / 3} \times 45.376^{-11 / 3} \times 559.200\right)^{3 / 5} \frac{M_{\odot}}{1.989 \times 10^{30}}=55.664 M_{\odot} \\
\mathscr{M}_{3}^{+}= & \frac{\left(2.998 \times 10^{8}\right)^{3}}{6.674 \times 10^{-11}}\left(\frac{5}{96} \pi^{-8 / 3} \times 58.445^{-11 / 3} \times 1142.134\right)^{3 / 5} \frac{M_{\odot}}{1.989 \times 10^{30}}=48.960 M_{\odot} \\
\mathscr{M}_{4}^{+}= & \frac{\left(2.998 \times 10^{8}\right)^{3}}{6.674 \times 10^{-11}}\left(\frac{5}{96} \pi^{-8 / 3} \times 79.318^{-11 / 3} \times 2747.764\right)^{3 / 5} \frac{M_{\odot}}{1.989 \times 10^{30}}=42.347 M_{\odot} \\
\mathscr{M}_{5}^{+}= & \frac{\left(2.998 \times 10^{8}\right)^{3}}{6.674 \times 10^{-11}}\left(\frac{5}{96} \pi^{-8 / 3} \times 116.644^{-11 / 3} \times 8657.494\right)^{3 / 5} \frac{M_{\odot}}{1.989 \times 10^{30}}=36.090 M_{\odot} \\
\mathscr{M}_{2}^{-}= & \frac{\left(2.998 \times 10^{8}\right)^{3}}{6.674 \times 10^{-11}}\left(\frac{5}{96} \pi^{-8 / 3} \times 42.731^{-11 / 3} \times 418.092\right)^{3 / 5} \frac{M_{\odot}}{1.989 \times 10^{30}}=53.356 M_{\odot} \\
\mathscr{M}_{3}^{-}= & \frac{\left(2.998 \times 10^{8}\right)^{3}}{6.674 \times 10^{-11}}\left(\frac{5}{96} \pi^{-8 / 3} \times 53.045537^{-11 / 3} \times 764.196\right)^{3 / 5} \frac{M_{\odot}}{1.989 \times 10^{30}}=47.616 M_{\odot} \\
\mathscr{M}_{4}^{-}= & \frac{\left(2.998 \times 10^{8}\right)^{3}}{6.674 \times 10^{-11}}\left(\frac{5}{96} \pi^{-8 / 3} \times 68.323^{-11 / 3} \times 1560.827\right)^{3 / 5} \frac{M_{\odot}}{1.989 \times 10^{30}}=41.881 M_{\odot} \\
\mathscr{M}_{5}^{-}= & \frac{\left(2.998 \times 10^{8}\right)^{3}}{6.674 \times 10^{-11}}\left(\frac{5}{96} \pi^{-8 / 3} \times 92.724^{-11 / 3} \times 3755.062\right)^{3 / 5} \frac{M_{\odot}}{1.989 \times 10^{30}}=36.224 M_{\odot} \\
\mathscr{M}_{6}^{-}= & \frac{\left(2.998 \times 10^{8}\right)^{3}}{6.674 \times 10^{-11}}\left(\frac{5}{96} \pi^{-8 / 3} \times 136.358^{-11 / 3} \times 11831.23\right)^{3 / 5} \frac{M_{\odot}}{1.989 \times 10^{30}}=30.873 M_{\odot}
\end{aligned}
$$

Among them, the positive and negative superscript indicates the calculation of positive and negative strains. The above approximate calculation results show that the frequency distribution of the positive and negative strain of the GW150914 signal wave determines two kinds of the chirp mass distribution of the wave source, which vary monotonically with the frequency of the change of the gravitational wave signal, and these values are very different. Before the merger, the rest mass of the two stars of the wave source of the GW150914 signal is invariable. Results of chirp mass estimation from different frequencies of the GW150914 signal wave are far from each other, and it is impossible to correct to be the approximate equivalent results. This shows that GW150914 signal wave does not support the general relativistic gravitational wave fre- quency equation.

Since the estimation of the chirp mass is derived from the zero order approximation of the general relativistic Blanchet frequency equation, the above difference may be interpreted as an error caused by the zero order approximation. It seems that there is no such obvious difference between the original Blanchet frequency equation and the observed value, or there will be no obvious difference between the advanced approximation of the Blanchet frequency equation and the observed value. Is the result calculated according to the exact Blanchet frequency equation or its high-order approximation highly consistent with the GW150914 signal? 


\section{Graphical proof of Relativistic Equation Failure for GW150914 Signal wave}

After detecting the gravitational wave signal and confirming that the wave source is a binary star gravitational system, the mass of the wave source can be determined by the frequency and strain distribution of the gravitational wave in theory. The Blanchet frequency equation is a highly nonlinear equation and cannot be solved accurately. However, in order to calculate the chirp mass, there is no scientific basis for choosing the zero order approximation of the equation under the low frequency condition. Ignoring the spin of the black hole, remove the spin-spin and spin-orbit interactions of the Blanchet frequency equation. The high-level approximation of the Blanchet equation is,

$$
\begin{aligned}
\pi \dot{f}= & \frac{96 G^{5 / 3} m_{1} m_{2}(\pi f)^{11 / 3}}{5 c^{5}\left(m_{1}+m_{2}\right)^{1 / 3}} \times\left\{1-\left[\frac{743}{336}+\frac{11 m_{1} m_{2}}{4\left(m_{1}+m_{2}\right)^{2}}\right]\left[\frac{G \pi f\left(m_{1}+m_{2}\right)}{c^{3}}\right]^{2 / 3}\right. \\
& \left.+4 \pi\left[\frac{G \pi f}{c^{3}}\left(m_{1}+m_{2}\right)\right]+\left[\frac{34103}{18144}+\frac{13661 m_{1} m_{2}}{2016\left(m_{1}+m_{2}\right)^{2}}+\frac{59\left(m_{1} m_{2}\right)^{2}}{18\left(m_{1}+m_{2}\right)^{4}}\right]\left[\frac{G \pi f}{c^{3}}\left(m_{1}+m_{2}\right)\right]^{4 / 3}\right\}
\end{aligned}
$$

Although this equation can not be solved accurately, the computer having developed to today, for some non-linear and implicit function equations which can not be solved accurately, approximate solutions with high accuracy can be obtained by numerical calculation or image solution to prove the reliability of qualitative conclusions. In this section, we prove that the relativistic Blanchet frequency equation of GW150914 signal wave is invalid by means of image solution.

\subsection{Incompatibility between theoretical and ex- perimental curves of GW150914 signal wave}

An image solution to Blanchet equation (5) is to first give different double black hole masses to the equation to draw various theoretical curves of frequency change rate and frequency relationship, and then draw the Lagrange frequency curve (i.e. experimental curve) representing the relationship between frequency change rate and frequency of GW150914 signal wave. If the latter coincides with one of the former or approaches, the mass of the wave source is closest to the mass assignment corresponding to this particular theoretical curve. Otherwise, if the experimental curve cannot coincide with or nearly coincide with any theoretical curve, it is proved that the Blanchet frequency equation has no signal wave solution of GW150914.

As shown in Figure 3, the broken line is the Lagrange frequency broken line (experimental curve LC) of the frequency change rate of GW150914 signal wave strain peak. In order to describe the relativistic Blanche frequency equation curve (theoretical curve BC) of GW150914 signal wave, seven groups of double star masses were selected, $m_{1}=29 m_{\odot}, m_{2}=36 m_{\odot} ; m_{1}=7 m_{\odot}, m_{2}=58 m_{\odot} ;$ $m_{1}=15 m_{\odot}, m_{2}=50 m_{\odot} ; m_{1}=20 m_{\odot}, m_{2}=45 m_{\odot} ;$ $m_{1}=10 m_{\odot}, m_{2}=55 m_{\odot} ; m_{1}=10 m_{\odot}, m_{2}=65 m_{\odot} ;$ $m_{1}=30 m_{\odot}, m_{2}=50 m_{\odot}$, in which $m_{\odot}=1.989 \times 10^{30} \mathrm{~kg}$ is the solar mass. Substituting the mass assignments of the above groups into equation (5) respectively, the following seven equations are obtained,

$$
\begin{aligned}
& \pi \dot{f}=\frac{96\left(6.674 \times 10^{-11}\right)^{5 / 3}\left(29 \times 1.989 \times 10^{30}\right)\left(36 \times 1.989 \times 10^{30}\right)(\pi \times f)^{11 / 3}}{5 \times\left(2.998 \times 10^{8}\right)^{5}\left(29 \times 1.989 \times 10^{30}+36 \times 1.989 \times 10^{30}\right)^{1 / 3}} \\
& \times\left\{1-\left(\frac{743}{336}+\frac{11 \times 29 \times 36}{4(29+36)^{2}}\right)\left(\frac{6.674 \times 10^{-11} \pi \times f \times(29+36) \times 1.989 \times 10^{30}}{\left(2.998 \times 10^{8}\right)^{3}}\right)^{2 / 3}\right. \\
& +4 \pi \times \frac{6.674 \times 10^{-11} \pi \times f \times(29+36) \times 1.989 \times 10^{30}}{\left(2.998 \times 10^{8}\right)^{3}} \\
& \left.+\left(\frac{34103}{18144}+\frac{13661 \times 29 \times 36}{2016(29+36)^{2}}+\frac{59 \times(29 \times 36)^{2}}{(29+36)^{4}}\right)\left(\frac{6.674 \times 10^{-11} \pi \times f \times(29+36) \times 1.989 \times 10^{30}}{\left(2.998 \times 10^{8}\right)^{3}}\right)^{4 / 3}\right\} \\
& \pi \dot{f}=\frac{96\left(6.674 \times 10^{-11}\right)^{5 / 3}\left(7 \times 1.989 \times 10^{30}\right)\left(58 \times 1.989 \times 10^{30}\right)(\pi \times f)^{11 / 3}}{5 \times\left(2.998 \times 10^{8}\right)^{5}\left(7 \times 1.989 \times 10^{30}+58 \times 1.989 \times 10^{30}\right)^{1 / 3}} \\
& \times\left\{1-\left(\frac{743}{336}+\frac{11 \times 7 \times 58}{4(7+58)^{2}}\right)\left(\frac{6.674 \times 10^{-11} \pi \times f \times(7+58) \times 1.989 \times 10^{30}}{\left(2.998 \times 10^{8}\right)^{3}}\right)^{2 / 3}\right. \\
& +4 \pi \times \frac{6.674 \times 10^{-11} \pi \times f \times(7+58) \times 1.989 \times 10^{30}}{\left(2.998 \times 10^{8}\right)^{3}}
\end{aligned}
$$




$$
\begin{aligned}
& \left.+\left(\frac{34103}{18144}+\frac{13661 \times 7 \times 58}{2016(7+58)^{2}}+\frac{59 \times(7 \times 58)^{2}}{(7+58)^{4}}\right)\left(\frac{6.674 \times 10^{-11} \pi \times f \times(7+58) \times 1.989 \times 10^{30}}{\left(2.998 \times 10^{8}\right)^{3}}\right)^{4 / 3}\right\} \\
& \pi \dot{f}=\frac{96\left(6.674 \times 10^{-11}\right)^{5 / 3}\left(15 \times 1.989 \times 10^{30}\right)\left(50 \times 1.989 \times 10^{30}\right)(\pi \times f)^{11 / 3}}{5 \times\left(2.998 \times 10^{8}\right)^{5}\left(15 \times 1.989 \times 10^{30}+50 \times 1.989 \times 10^{30}\right)^{1 / 3}} \\
& \times\left\{1-\left(\frac{743}{336}+\frac{11 \times 15 \times 50}{4(15+50)^{2}}\right)\left(\frac{6.674 \times 10^{-11} \pi \times f \times(15+50) \times 1.989 \times 10^{30}}{\left(2.998 \times 10^{8}\right)^{3}}\right)^{2 / 3}\right. \\
& +4 \pi \times \frac{6.674 \times 10^{-11} \pi \times f \times(15+50) \times 1.989 \times 10^{30}}{\left(2.998 \times 10^{8}\right)^{3}} \\
& \left.+\left(\frac{34103}{18144}+\frac{13661 \times 15 \times 50}{2016(15+50)^{2}}+\frac{59 \times(15 \times 50)^{2}}{(15+50)^{4}}\right)\left(\frac{6.674 \times 10^{-11} \pi \times f \times(15+50) \times 1.989 \times 10^{30}}{\left(2.998 \times 10^{8}\right)^{3}}\right)^{4 / 3}\right\} \\
& \pi \dot{f}=\frac{96\left(6.674 \times 10^{-11}\right)^{5 / 3}\left(25 \times 1.989 \times 10^{30}\right)\left(40 \times 1.989 \times 10^{30}\right)(\pi \times f)^{11 / 3}}{5 \times\left(2.998 \times 10^{8}\right)^{5}\left(25 \times 1.989 \times 10^{30}+40 \times 1.989 \times 10^{30}\right)^{1 / 3}} \\
& \times\left\{1-\left(\frac{743}{336}+\frac{11 \times 25 \times 40}{4(25+40)^{2}}\right)\left(\frac{6.674 \times 10^{-11} \pi \times f \times(25+40) \times 1.989 \times 10^{30}}{\left(2.998 \times 10^{8}\right)^{3}}\right)^{2 / 3}\right. \\
& +4 \pi \times \frac{6.674 \times 10^{-11} \pi \times f \times(25+40) \times 1.989 \times 10^{30}}{\left(2.998 \times 10^{8}\right)^{3}} \\
& \left.+\left(\frac{34103}{18144}+\frac{13661 \times 25 \times 40}{2016(25+40)^{2}}+\frac{59 \times(25 \times 40)^{2}}{(25+40)^{4}}\right)\left(\frac{6.674 \times 10^{-11} \pi \times f \times(25+40) \times 1.989 \times 10^{30}}{\left(2.998 \times 10^{8}\right)^{3}}\right)^{4 / 3}\right\} \\
& \pi \dot{f}=\frac{96\left(6.674 \times 10^{-11}\right)^{5 / 3}\left(10 \times 1.989 \times 10^{30}\right)\left(55 \times 1.989 \times 10^{30}\right)(\pi \times f)^{11 / 3}}{5 \times\left(2.998 \times 10^{8}\right)^{5}\left(10 \times 1.989 \times 10^{30}+55 \times 1.989 \times 10^{30}\right)^{1 / 3}} \\
& \times\left\{1-\left(\frac{743}{336}+\frac{11 \times 10 \times 55}{4(10+55)^{2}}\right)\left(\frac{6.674 \times 10^{-11} \pi \times f \times(10+55) \times 1.989 \times 10^{30}}{\left(2.998 \times 10^{8}\right)^{3}}\right)^{2 / 3}\right. \\
& +4 \pi \times \frac{6.674 \times 10^{-11} \pi \times f \times(10+55) \times 1.989 \times 10^{30}}{\left(2.998 \times 10^{8}\right)^{3}} \\
& \left.+\left(\frac{34103}{18144}+\frac{13661 \times 10 \times 55}{2016(10+55)^{2}}+\frac{59 \times(10 \times 55)^{2}}{(10+55)^{4}}\right)\left(\frac{6.674 \times 10^{-11} \pi \times f \times(10+55) \times 1.989 \times 10^{30}}{\left(2.998 \times 10^{8}\right)^{3}}\right)^{4 / 3}\right\} \\
& \pi \dot{f}=\frac{96\left(6.674 \times 10^{-11}\right)^{5 / 3}\left(10 \times 1.989 \times 10^{30}\right)\left(65 \times 1.989 \times 10^{30}\right)(\pi \times f)^{11 / 3}}{5 \times\left(2.998 \times 10^{8}\right)^{5}\left(10 \times 1.989 \times 10^{30}+65 \times 1.989 \times 10^{30}\right)^{1 / 3}} \\
& \times\left\{1-\left(\frac{743}{336}+\frac{11 \times 10 \times 65}{4(10+65)^{2}}\right)\left(\frac{6.674 \times 10^{-11} \pi \times f \times(10+65) \times 1.989 \times 10^{30}}{\left(2.998 \times 10^{8}\right)^{3}}\right)^{2 / 3}\right. \\
& +4 \pi \times \frac{6.674 \times 10^{-11} \pi \times f \times(10+65) \times 1.989 \times 10^{30}}{\left(2.998 \times 10^{8}\right)^{3}} \\
& \left.+\left(\frac{34103}{18144}+\frac{13661 \times 10 \times 65}{2016(10+65)^{2}}+\frac{59 \times(10 \times 65)^{2}}{(10+65)^{4}}\right)\left(\frac{6.674 \times 10^{-11} \pi \times f \times(10+65) \times 1.989 \times 10^{30}}{\left(2.998 \times 10^{8}\right)^{3}}\right)^{4 / 3}\right\} \\
& \pi \dot{f}=\frac{96\left(6.674 \times 10^{-11}\right)^{5 / 3}\left(30 \times 1.989 \times 10^{30}\right)\left(50 \times 1.989 \times 10^{30}\right)(\pi \times f)^{11 / 3}}{5 \times\left(2.998 \times 10^{8}\right)^{5}\left(30 \times 1.989 \times 10^{30}+50 \times 1.989 \times 10^{30}\right)^{1 / 3}} \\
& \times\left\{1-\left(\frac{743}{336}+\frac{11 \times 30 \times 50}{4(30+50)^{2}}\right)\left(\frac{6.674 \times 10^{-11} \pi \times f \times(30+50) \times 1.989 \times 10^{30}}{\left(2.998 \times 10^{8}\right)^{3}}\right)^{2 / 3}\right. \\
& +4 \pi \times \frac{6.674 \times 10^{-11} \pi \times f \times(30+50) \times 1.989 \times 10^{30}}{\left(2.998 \times 10^{8}\right)^{3}} \\
& \left.+\left(\frac{34103}{18144}+\frac{13661 \times 30 \times 50}{2016(30+50)^{2}}+\frac{59 \times(30 \times 50)^{2}}{(30+50)^{4}}\right)\left(\frac{6.674 \times 10^{-11} \pi \times f \times(30+50) \times 1.989 \times 10^{30}}{\left(2.998 \times 10^{8}\right)^{3}}\right)^{4 / 3}\right\}
\end{aligned}
$$




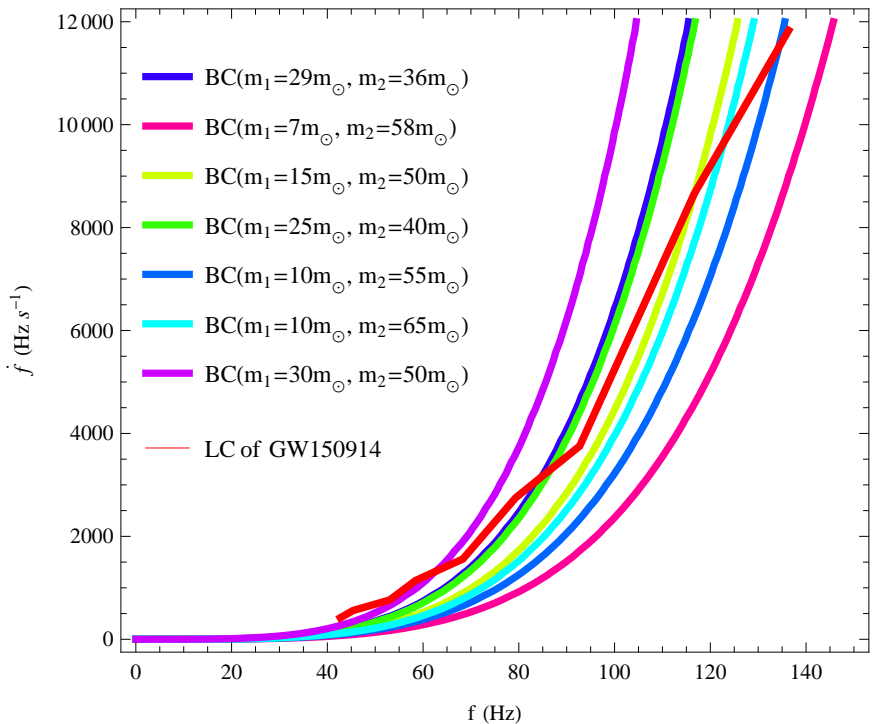

Figure 3 Lagrange frequency curve (experimental curve LC) and Blanchet frequency curve (theoretical curve BC) of GW150914 signal wave. Graph lines intuitively reflect that the slope of each Blanchet frequency curve changes rapidly with increasing frequency, and the Lagrange frequency polyline cannot coincide with a certain Blancchet frequency curve, indicating that the GW150914 signal does not support the generally relativistic Blanchet equation.

These seven Blanchet frequency curves (BC) are also plotted in Figure 3. Theoretically, the Lagrange frequency polyline of the signal wave should be approximately coincident with the Blanchet frequency curve of the $36 M_{\odot}$ and $29 M_{\odot}$ mass combinations. Therefore, the masses of the two black holes of the wave source can be found the best approximation from the curve. However, the fact is that the Lagrange polylines of the GW150914 signal waves deviate far from the Blanchet frequency curve of gravitational waves from binary black holes with masses of $36 M_{\odot}$ and $29 M_{\odot}$, which is contradictory! More importantly, the Lagrange polyline of the GW150914 signal wave cuts the Blanchet curves of different mass combinations. This shows that there is no mass assignment of any group of binary stars to make the Blanchet frequency curve coincide with the Lagrange frequency polyline of the GW150914 signal wave.

Taking the combined values of different binary star masses $m_{1}$ and $m_{2}$ into the Blanche frequency equation can draw a lot of Blancchet frequency curves. When the total mass is kept constant, the masses of the binary stars are closer, and the Blanchet frequency curve is steeper with increasing frequency. The trend of all these Blanchet curves and Lagrange frequency polylines predicts that the Lagrange polyline must cut all the Blanchet curves. It is also impossible to modify the vibration curve of the GW150914 signal wave so that its frequency distribution satisfies the Blanchet frequency equation.

Therefore, the frequency variation law of gw150914 signal wave does not satisfy the relativistic Blanchet frequency equation. It is lack of the minimum standard of scientific proof to declare that gw150914 signal wave comes from the merging process of double black holes with 36 and 29 solar masses.

\subsection{Unsolvability of Blanchet equations for G- W150914 signal}

The Lagrange frequency polyline of the GW150914 signal wave cutting the Blanchet frequency curve of different mass combinations shows that the Blanchet frequency equation does not have any solution that satisfies the GW150914 signal wave. This conclusion can also be rigorously proved by another image solution.

Substituting the frequencies of the positive and negative strain peaks in Table 1 and their Lagrange derivatives into the Blanche frequency equation (5) in order, a system of nine Blanchet equations is obtained,

$$
\begin{aligned}
\pi \times & 559.1999942=\frac{96\left(6.674 \times 10^{-11}\right)^{5 / 3}\left(m_{1} \times 1.989 \times 10^{30}\right)\left(m_{2} \times 1.989 \times 10^{30}\right)(\pi \times 45.37639637)^{11 / 3}}{5 \times\left(2.998 \times 10^{8}\right)^{5}\left(m_{1} \times 1.989 \times 10^{30}+m_{2} \times 1.989 \times 10^{30}\right)^{1 / 3}} \\
& \times\left\{1-\left(\frac{743}{336}+\frac{11 m_{1} \times m_{2}}{4\left(m_{1}+m_{2}\right)^{2}}\right)\left(\frac{6.674 \times 10^{-11} \pi \times 45.37639637\left(m_{1}+m_{2}\right) \times 1.989 \times 10^{30}}{\left(2.998 \times 10^{8}\right)^{3}}\right)^{2 / 3}\right. \\
& +4 \pi \times \frac{6.674 \times 10^{-11} \pi \times 45.37639637\left(m_{1}+m_{2}\right) \times 1.989 \times 10^{30}}{\left(2.998 \times 10^{8}\right)^{3}} \\
& \left.+\left(\frac{34103}{18144}+\frac{13661 m_{1} m_{2}}{2016\left(m_{1}+m_{2}\right)^{2}}+\frac{59\left(m_{1} m_{2}\right)^{2}}{\left(m_{1}+m_{2}\right)^{4}}\right) \times\left(\frac{6.674 \times 10^{-11} \pi \times 45.37639637\left(m_{1}+m_{2}\right) \times 1.989 \times 10^{30}}{\left(2.998 \times 10^{8}\right)^{3}}\right)^{4 / 3}\right\} \\
\pi \times & 1142.134177=\frac{96\left(6.674 \times 10^{-11}\right)^{5 / 3}\left(m_{1} \times 1.989 \times 10^{30}\right)\left(m_{2} \times 1.989 \times 10^{30}\right)(\pi \times 58.44479852)^{11 / 3}}{5 \times\left(2.998 \times 10^{8}\right)^{5}\left(m_{1} \times 1.989 \times 10^{30}+m_{2} \times 1.989 \times 10^{30}\right)^{1 / 3}} \\
& \times\left\{1-\left(\frac{743}{336}+\frac{11 m_{1} m_{2}}{4\left(m_{1}+m_{2}\right)^{2}}\right)\left(\frac{6.674 \times 10^{-11} \pi \times 58.44479852\left(m_{1}+m_{2}\right) \times 1.989 \times 10^{30}}{\left(2.998 \times 10^{8}\right)^{3}}\right)^{2 / 3}\right. \\
& +4 \pi \times \frac{6.674 \times 10^{-11} \pi \times 58.44479852\left(m_{1}+m_{2}\right) \times 1.989 \times 10^{30}}{\left(2.998 \times 10^{8}\right)^{3}}
\end{aligned}
$$




$$
\begin{aligned}
& \left.+\left(\frac{34103}{18144}+\frac{13661 m_{1} m_{2}}{2016\left(m_{1}+m_{2}\right)^{2}}+\frac{59\left(m_{1} m_{2}\right)^{2}}{\left(m_{1}+m_{2}\right)^{4}}\right)\left(\frac{6.674 \times 10^{-11} \pi \times 58.44479852\left(m_{1}+m_{2}\right) \times 1.989 \times 10^{30}}{\left(2.998 \times 10^{8}\right)^{3}}\right)^{4 / 3}\right\} \\
& \pi \times 2747.763841=\frac{96\left(6.674 \times 10^{-11}\right)^{5 / 3}\left(m_{1} \times 1.989 \times 10^{30}\right)\left(m_{2} \times 1.989 \times 10^{30}\right)(\pi \times 79.31794085)^{11 / 3}}{5 \times\left(2.998 \times 10^{8}\right)^{5}\left(m_{1} \times 1.989 \times 10^{30}+m_{2} \times 1.989 \times 10^{30}\right)^{1 / 3}} \\
& \times\left\{1-\left(\frac{743}{336}+\frac{11 m_{1} m_{2}}{4\left(m_{1}+m_{2}\right)^{2}}\right)\left(\frac{6.674 \times 10^{-11} \pi \times 79.31794085\left(m_{1}+m_{2}\right) \times 1.989 \times 10^{30}}{\left(2.998 \times 10^{8}\right)^{3}}\right)^{2 / 3}\right. \\
& +4 \pi \times \frac{6.674 \times 10^{-11} \pi \times 79.31794085\left(m_{1}+m_{2}\right) \times 1.989 \times 10^{30}}{\left(2.998 \times 10^{8}\right)^{3}} \\
& \left.+\left(\frac{34103}{18144}+\frac{13661 m_{1} m_{2}}{2016\left(m_{1}+m_{2}\right)^{2}}+\frac{59\left(m_{1} m_{2}\right)^{2}}{\left(m_{1}+m_{2}\right)^{4}}\right)\left(\frac{6.674 \times 10^{-11} \pi \times 79.31794085\left(m_{1}+m_{2}\right) \times 1.989 \times 10^{30}}{\left(2.998 \times 10^{8}\right)^{3}}\right)^{4 / 3}\right\} \\
& \pi \times 8657.49422=\frac{96\left(6.674 \times 10^{-11}\right)^{5 / 3}\left(m_{1} \times 1.989 \times 10^{30}\right)\left(m_{2} \times 1.989 \times 10^{30}\right)(\pi \times 116.6440307)^{11 / 3}}{5 \times\left(2.998 \times 10^{8}\right)^{5}\left(m_{1} \times 1.989 \times 10^{30}+m_{2} \times 1.989 \times 10^{30}\right)^{1 / 3}} \\
& \times\left\{1-\left(\frac{743}{336}+\frac{11 m_{1} m_{2}}{4\left(m_{1}+m_{2}\right)^{2}}\right)\left(\frac{6.674 \times 10^{-11} \pi \times 116.6440307\left(m_{1}+m_{2}\right) \times 1.989 \times 10^{30}}{\left(2.998 \times 10^{8}\right)^{3}}\right)^{2 / 3}\right. \\
& +4 \pi \times \frac{6.674 \times 10^{-11} \pi \times 116.6440307\left(m_{1}+m_{2}\right) \times 1.989 \times 10^{30}}{\left(2.998 \times 10^{8}\right)^{3}} \\
& \left.+\left(\frac{34103}{18144}+\frac{13661 m_{1} m_{2}}{2016\left(m_{1}+m_{2}\right)^{2}}+\frac{59\left(m_{1} m_{2}\right)^{2}}{\left(m_{1}+m_{2}\right)^{4}}\right)\left(\frac{6.674 \times 10^{-11} \pi \times 116.6440307\left(m_{1}+m_{2}\right) \times 1.989 \times 10^{30}}{\left(2.998 \times 10^{8}\right)^{3}}\right)^{4 / 3}\right\} \\
& \pi \times 418.0919129=\frac{96\left(6.674 \times 10^{-11}\right)^{5 / 3}\left(m_{1} \times 1.989 \times 10^{30}\right)\left(m_{2} \times 1.989 \times 10^{30}\right)(\pi \times 42.73112738)^{11 / 3}}{5 \times\left(2.998 \times 10^{8}\right)^{5}\left(m_{1} \times 1.989 \times 10^{30}+m_{2} \times 1.989 \times 10^{30}\right)^{1 / 3}} \\
& \times\left\{1-\left(\frac{743}{336}+\frac{11 m_{1} m_{2}}{4\left(m_{1}+m_{2}\right)^{2}}\right)\left(\frac{6.674 \times 10^{-11} \pi \times 42.73112738\left(m_{1}+m_{2}\right) \times 1.989 \times 10^{30}}{\left(2.998 \times 10^{8}\right)^{3}}\right)^{2 / 3}\right. \\
& +4 \pi \times \frac{6.674 \times 10^{-11} \pi \times 42.73112738\left(m_{1}+m_{2}\right) \times 1.989 \times 10^{30}}{\left(2.998 \times 10^{8}\right)^{3}} \\
& \left.+\left(\frac{34103}{18144}+\frac{13661 m_{1} m_{2}}{2016\left(m_{1}+m_{2}\right)^{2}}+\frac{59\left(m_{1} m_{2}\right)^{2}}{\left(m_{1}+m_{2}\right)^{4}}\right)\left(\frac{6.674 \times 10^{-11} \pi \times 42.73112738\left(m_{1}+m_{2}\right) \times 1.989 \times 10^{30}}{\left(2.998 \times 10^{8}\right)^{3}}\right)^{4 / 3}\right\} \\
& \pi \times 764.1961764=\frac{96\left(6.674 \times 10^{-11}\right)^{5 / 3}\left(m_{1} \times 1.989 \times 10^{30}\right)\left(m_{2} \times 1.989 \times 10^{30}\right)(\pi \times 53.04553744)^{11 / 3}}{5 \times\left(2.998 \times 10^{8}\right)^{5}\left(m_{1} \times 1.989 \times 10^{30}+m_{2} \times 1.989 \times 10^{30}\right)^{1 / 3}} \\
& \times\left\{1-\left(\frac{743}{336}+\frac{11 m_{1} m_{2}}{4\left(m_{1}+m_{2}\right)^{2}}\right)\left(\frac{6.674 \times 10^{-11} \pi \times 53.04553744\left(m_{1}+m_{2}\right) \times 1.989 \times 10^{30}}{\left(2.998 \times 10^{8}\right)^{3}}\right)^{2 / 3}\right. \\
& +4 \pi \times \frac{6.674 \times 10^{-11} \pi \times 53.04553744\left(m_{1}+m_{2}\right) \times 1.989 \times 10^{30}}{\left(2.998 \times 10^{8}\right)^{3}} \\
& \left.+\left(\frac{34103}{18144}+\frac{13661 m_{1} m_{2}}{2016\left(m_{1}+m_{2}\right)^{2}}+\frac{59\left(m_{1} m_{2}\right)^{2}}{\left(m_{1}+m_{2}\right)^{4}}\right)\left(\frac{6.674 \times 10^{-11} \pi \times 53.04553744\left(m_{1}+m_{2}\right) \times 1.989 \times 10^{30}}{\left(2.998 \times 10^{8}\right)^{3}}\right)^{4 / 3}\right\} \\
& \pi \times 1560.827218=\frac{96\left(6.674 \times 10^{-11}\right)^{5 / 3}\left(m_{1} \times 1.989 \times 10^{30}\right)\left(m_{2} \times 1.989 \times 10^{30}\right)(\pi \times 68.32265222)^{11 / 3}}{5 \times\left(2.998 \times 10^{8}\right)^{5}\left(m_{1} \times 1.989 \times 10^{30}+m_{2} \times 1.989 \times 10^{30}\right)^{1 / 3}} \\
& \times\left\{1-\left(\frac{743}{336}+\frac{11 m_{1} m_{2}}{4\left(m_{1}+m_{2}\right)^{2}}\right)\left(\frac{6.674 \times 10^{-11} \pi \times 68.32265222\left(m_{1}+m_{2}\right) \times 1.989 \times 10^{30}}{\left(2.998 \times 10^{8}\right)^{3}}\right)^{2 / 3}\right. \\
& +4 \pi \times \frac{6.674 \times 10^{-11} \pi \times 68.32265222\left(m_{1}+m_{2}\right) \times 1.989 \times 10^{30}}{\left(2.998 \times 10^{8}\right)^{3}} \\
& \left.+\left(\frac{34103}{18144}+\frac{13661 m_{1} m_{2}}{2016\left(m_{1}+m_{2}\right)^{2}}+\frac{59\left(m_{1} m_{2}\right)^{2}}{\left(m_{1}+m_{2}\right)^{4}}\right)\left(\frac{6.674 \times 10^{-11} \pi \times 68.32265222\left(m_{1}+m_{2}\right) \times 1.989 \times 10^{30}}{\left(2.998 \times 10^{8}\right)^{3}}\right)^{4 / 3}\right\}
\end{aligned}
$$




$$
\begin{aligned}
\pi \times & 3755.061951=\frac{96\left(6.674 \times 10^{-11}\right)^{5 / 3}\left(m_{1} \times 1.989 \times 10^{30}\right)\left(m_{2} \times 1.989 \times 10^{30}\right)(\pi \times 92.72359945)^{11 / 3}}{5 \times\left(2.998 \times 10^{8}\right)^{5}\left(m_{1} \times 1.989 \times 10^{30}+m_{2} \times 1.989 \times 10^{30}\right)^{1 / 3}} \\
& \times\left\{1-\left(\frac{743}{336}+\frac{11 m_{1} m_{2}}{4\left(m_{1}+m_{2}\right)^{2}}\right)\left(\frac{6.674 \times 10^{-11} \pi \times 92.72359945\left(m_{1}+m_{2}\right) \times 1.989 \times 10^{30}}{\left(2.998 \times 10^{8}\right)^{3}}\right)^{2 / 3}\right. \\
& +4 \pi \times \frac{6.674 \times 10^{-11} \pi \times 92.72359945\left(m_{1}+m_{2}\right) \times 1.989 \times 10^{30}}{\left(2.998 \times 10^{8}\right)^{3}} \\
& \left.+\left(\frac{34103}{18144}+\frac{13661 m_{1} m_{2}}{2016\left(m_{1}+m_{2}\right)^{2}}+\frac{59\left(m_{1} m_{2}\right)^{2}}{\left(m_{1}+m_{2}\right)^{4}}\right)\left(\frac{6.674 \times 10^{-11} \pi \times 92.72359945\left(m_{1}+m_{2}\right) \times 1.989 \times 10^{30}}{\left(2.998 \times 10^{8}\right)^{3}}\right)^{4 / 3}\right\} \\
\pi \times & 11831.23042=\frac{96\left(6.674 \times 10^{-11}\right)^{5 / 3}\left(m_{1} \times 1.989 \times 10^{30}\right)\left(m_{2} \times 1.989 \times 10^{30}\right)(\pi \times 136.3582345)^{11 / 3}}{5 \times\left(2.998 \times 10^{8}\right)^{5}\left(m_{1} \times 1.989 \times 10^{30}+m_{2} \times 1.989 \times 10^{30}\right)^{1 / 3}} \\
& \times\left\{1-\left(\frac{743}{336}+\frac{11 m_{1} m_{2}}{4\left(m_{1}+m_{2}\right)^{2}}\right)\left(\frac{6.674 \times 10^{-11} \pi \times 136.3582345\left(m_{1}+m_{2}\right) \times 1.989 \times 10^{30}}{\left(2.998 \times 10^{8}\right)^{3}}\right)^{2 / 3}\right. \\
& +4 \pi \times \frac{6.674 \times 10^{-11} \pi \times 136.3582345\left(m_{1}+m_{2}\right) \times 1.989 \times 10^{30}}{\left(2.998 \times 10^{8}\right)^{3}} \\
& \left.+\left(\frac{34103}{18144}+\frac{13661 m_{1} m_{2}}{2016\left(m_{1}+m_{2}\right)^{2}}+\frac{59\left(m_{1} m_{2}\right)^{2}}{\left(m_{1}+m_{2}\right)^{4}}\right)\left(\frac{6.674 \times 10^{-11} \pi \times 136.3582345\left(m_{1}+m_{2}\right) \times 1.989 \times 10^{30}}{\left(2.998 \times 10^{8}\right)^{3}}\right)^{4 / 3}\right\}
\end{aligned}
$$

Theoretically, the solution of this system of equations must exist and be unique. Otherwise, the result will prove that the Blanchet frequency equation does not have a GW150914 signal wave solution. The unknowns of the system of equations have only two masses, $m_{1}$ and $m_{2}$, and the positions of the two can be exchanged. The curves of these equations are plotted. According to the image solution, these curves should intersect in two very small regions within the error range. The coordinates correspond to the mass of the two black holes of the source. However, as shown in Fig. 4, similar to the isotherm of the ideal gas in the closed container, the nine Blanchet curves corresponding to the frequency and corresponding time derivative of the signal wave strain peak of the GW150914 signal wave are disjoint, and the results negate the existence and uniqueness of the solution to the equation, that is, the GW150914 signal wave dos not support the Blanchet frequency equation.

The image solution can explain intuitively and concisely whether the specified signal wave solution of the non-linear Blanchet equation exists or is unique. In fact, computer graphics can also give high-precision numerical solutions. Therefore, the image solution is one of the best method$\mathrm{s}$ for dealing with gravitational wave detection data and solving nonlinear Blancht frequency equations. Observations of the GW150914 signal wave are inconsistent with the results of the image solution of the Blanchet frequency equation, which is a general relativity inference, and there is a big difference between the general relativity prediction and the real gravitational wave signal. This is the reason why a reasonable result cannot be determined by using the Blanche frequency equation to estimate the chirp mass of the GW150914 signal source.

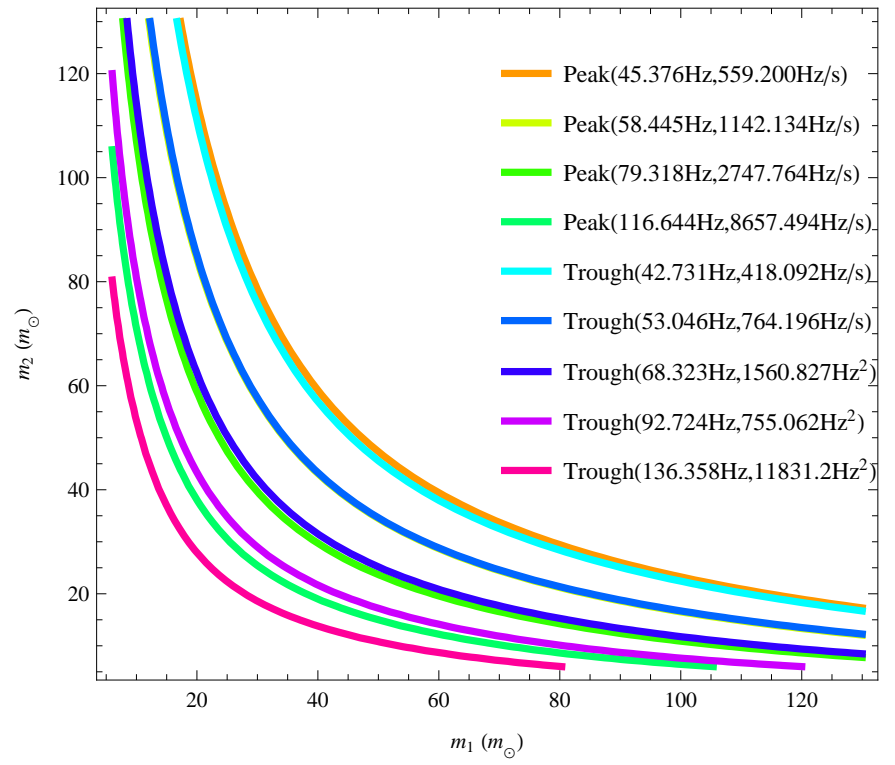

Figure 4 The Blanchet mass curves of the GW150914 signal wave. The group of curves is similar to the isotherm without two intersection regions, showing that the Blanchet frequency equation has no a GW150914 signal wave solution.

In summary, if the GW150914 signal wave comes from the merger of two spiral black holes with masses of 29 and 36 solar masses respectively, then the Lagrange frequency polyline of the GW150914 signal wave will inevitably coincide with the Blanchet frequency curve. However, the Lagrange frequency polyline of the GW150914 signal wave cuts all the Blanchet frequency curves of wave source mass combinations including 29 and 36 solar masses, so the Blanchet frequency equation does not have a GW150914 signal wave solution. However, the Lagrange frequency polyline of the GW150914 signal wave cuts al$l$ the Blanchet frequency curves of different wave source 
mass combinations including 29 and 36 solar masses, so the Blanchet frequency equation does not have a GW150914 signal wave solution. The Blanchet frequency equation set determined by the frequency and its time derivative contains only the mass parameters, but the Blanch curve corresponding to the strain peak frequency of the GW150914 signal wave is discrete without intersections, which also indicates that the Blanchet frequency equation does not have a GW150914 signal wave solution. There are indelible essential differences between the GW150914 signal wave and the general relativistic Blanchet frequency equation.

Among all LIGO signals, only GW50914 signal has obvious monotonic change characteristics, and accurate data record can be used for quantitative analysis. It is concluded that the relativistic equation of LIGO signal is invalid, or specifically, the Blanchet frequency equation of LIGO signal is invalid.

\section{Uncertainty of chirp mass of numerical rel- ativistic waveform}

There is a difference between the frequency distribution law of the GW150914 signal wave and the general rela- tivity Blanket frequency equation. Let us now study the frequency distribution law of the so-called numerical relativistic waveform of the GW150914 signal wave drawn by LIGO to understand the credibility of the numerical relativistic waveform. As shown in Fig. 5, the time of the positive and negative strain peaks of the numerical relativistic waveform of LIGO is first extracted, and the corresponding periods, frequencies, and frequency change rates are calculated. All the results are listed in Table 3. It is shown from the calculation results that the $\dot{f}_{i} \dot{f}_{i}^{-2}$ values of the positive strain peaks and the negative strain peaks of the numerical relativistic waveform do not have the same distribution, which deviates from the law that the $\dot{f}_{i} \dot{f}_{i}^{-2}$ values of the positive strain peaks and the negative strain peaks of the original GW150914 waveforms have the same distribution. On the other hand, the $\dot{f}_{i} \dot{f}_{i}^{-11 / 3}$ value of the numerical relativistic waveform is also not a constant approximated by the zero-order approximation (3) of the Blanchet's frequency equation, and the value of $\dot{f}_{i} \dot{f}_{i}^{-11 / 3}$ at high frequencies is very different in particular. It can be seen that the drawing of the numerical relativistic waveform of GW150914 does not meet the requirement of logic self-consistent.

Table 3 The positive and negative strain frequencies and their change rates of the numerical relativistic waveform

\begin{tabular}{|c|c|c|c|c|c|c|c|c|c|c|}
\hline \multirow{2}{*}{$\mathrm{i}$} & \multicolumn{5}{|c|}{ Positive strain of LIGO relativistic waveform } & \multicolumn{5}{|c|}{ Negative strain of LIGO relativistic waveform } \\
\hline & $t_{i}$ & $f_{i}$ & $\dot{f}_{i}$ & $\dot{f}_{i} / f_{i}^{2}$ & $\dot{f}_{i} / f_{i}^{11 / 3}$ & $t_{i}$ & $f_{i}$ & $\dot{f}_{i}$ & $\dot{f}_{i} / f_{i}^{2}$ & $\dot{f}_{i} / f_{i}^{11 / 3}$ \\
\hline 1 & 0.2805 & & & & & 0.2644 & & & & \\
\hline 2 & 0.3103 & 33.55705 & & & & 0.2955 & & & & \\
\hline 3 & 0.3377 & 36.49635 & 132.99008 & 0.09984 & 0.00024864 & 0.3243 & 32.15434 & & & \\
\hline 4 & 0.3624 & 40.48583 & 216.78090 & 0.13226 & 0.00027706 & 0.3507 & 34.72222 & 103.70375 & 0.08602 & 0.00023276 \\
\hline 5 & 0.3839 & 46.51163 & 399.33703 & 0.18459 & 0.00030687 & 0.3737 & 37.87879 & 177.24775 & 0.12353 & 0.00028915 \\
\hline 6 & 0.4017 & 56.17978 & 901.12942 & 0.28551 & 0.00034647 & 0.3934 & 43.47826 & 301.70102 & 0.15960 & 0.00029688 \\
\hline 7 & 0.4151 & 74.62687 & 3215.89835 & 0.57745 & 0.00043654 & 0.409 & 50.76142 & 584.25788 & 0.22674 & 0.00032582 \\
\hline 8 & 0.4231 & 125.0000 & 9644.08726 & 0.61722 & 0.00019751 & 0.4195 & 64.10256 & 1704.08712 & 0.41471 & 0.00040391 \\
\hline 9 & 0.4281 & 200.0000 & 10880.0774 & 0.27200 & 0.00003977 & 0.4259 & 95.23810 & 5452.51100 & 0.60114 & 0.00030266 \\
\hline 10 & 0.4325 & 227.2727 & 5164.99283 & 0.09999 & 0.00001181 & 0.4306 & 156.2500 & 10588.0957 & 0.43369 & 0.00009568 \\
\hline 11 & 0.4366 & 243.9024 & & & & 0.4346 & 212.7660 & 10775.8621 & 0.23804 & 0.00003139 \\
\hline
\end{tabular}

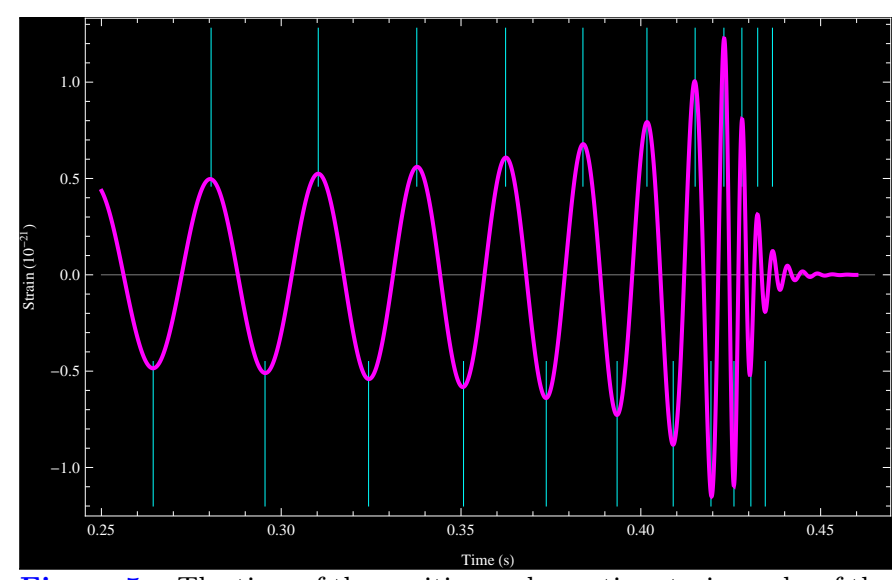

Figure 5 The time of the positive and negative strain peaks of the numerical relativistic waveform of the GW150914 signal wave $^{[21]}$.

The specific operational procedure of the numerical rel- ativistic waveform has not been disclosed, and the real physical meaning has not caused the attention it deserves. The GW150914 signal wave was identified as coming from a far-ancient spectacle of the merger of spiral binary black holes with $29 M_{\odot}$ and $36 M_{\odot}$ respectively, and mass of the combined black hole is $62 M_{\odot}$. How to draw such a conclusion, the calculation process of argument is missing. However, it is one of the key procedures to test the theory of relativistic gravitational waves. Now we use the so-called numerical relativistic gravitational waveform of the GW150914 signal wave to estimate the chirp mass of the wave source. According to the values in Table 3, the peaks and troughs of the numerical relativistic waveform shown in Figure 5 have eight Lagrange frequency derivatives, respectively, which are substituted into the approximate equation (4) to estimate the chirp mass. The result has 16 different values, 


$$
\begin{aligned}
& \mathscr{M}_{3}^{+}=\frac{\left(2.998 \times 10^{8}\right)^{3}}{6.674 \times 10^{-11}}\left(\frac{5}{96} \pi^{-8 / 3} \times 36.49635^{-11 / 3} \times 132.99008\right)^{3 / 5} \frac{M_{\odot}}{1.989 \times 10^{30}}=37.9661 M_{\odot} \\
& \mathscr{M}_{4}^{+}=\frac{\left(2.998 \times 10^{8}\right)^{3}}{6.674 \times 10^{-11}}\left(\frac{5}{96} \pi^{-8 / 3} \times 40.48583^{-11 / 3} \times 216.78090\right)^{3 / 5} \frac{M_{\odot}}{1.989 \times 10^{30}}=43.0746 M_{\odot} \\
& \mathscr{M}_{5}^{+}=\frac{\left(2.998 \times 10^{8}\right)^{3}}{6.674 \times 10^{-11}}\left(\frac{5}{96} \pi^{-8 / 3} \times 46.51163^{-11 / 3} \times 399.33703\right)^{3 / 5} \frac{M_{\odot}}{1.989 \times 10^{30}}=43.0746 M_{\odot} \\
& \mathscr{M}_{6}^{+}=\frac{\left(2.998 \times 10^{8}\right)^{3}}{6.674 \times 10^{-11}}\left(\frac{5}{96} \pi^{-8 / 3} \times 56.17978^{-11 / 3} \times 901.12942\right)^{3 / 5} \frac{M_{\odot}}{1.989 \times 10^{30}}=46.3286 M_{\odot} \\
& \mathscr{M}_{7}^{+}=\frac{\left(2.998 \times 10^{8}\right)^{3}}{6.674 \times 10^{-11}}\left(\frac{5}{96} \pi^{-8 / 3} \times 74.62687^{-11 / 3} \times 3215.89835\right)^{3 / 5} \frac{M_{\odot}}{1.989 \times 10^{30}}=53.2188 M_{\odot} \\
& \mathscr{M}_{8}^{+}=\frac{\left(2.998 \times 10^{8}\right)^{3}}{6.674 \times 10^{-11}}\left(\frac{5}{96} \pi^{-8 / 3} \times 125.00000^{-11 / 3} \times 9644.08726\right)^{3 / 5} \frac{M_{\odot}}{1.989 \times 10^{30}}=33.0680 M_{\odot} \\
& \mathscr{M}_{9}^{+}=\frac{\left(2.998 \times 10^{8}\right)^{3}}{6.674 \times 10^{-11}}\left(\frac{5}{96} \pi^{-8 / 3} \times 200.00000^{-11 / 3} \times 10880.07737\right)^{3 / 5} \frac{M_{\odot}}{1.989 \times 10^{30}}=12.6406 M_{\odot} \\
& \mathscr{M}_{10}^{+}=\frac{\left(2.998 \times 10^{8}\right)^{3}}{6.674 \times 10^{-11}}\left(\frac{5}{96} \pi^{-8 / 3} \times 227.27270^{-11 / 3} \times 5164.99283\right)^{3 / 5} \frac{M_{\odot}}{1.989 \times 10^{30}}=6.1023 M_{\odot} \\
& \mathscr{M}_{3}^{-}=\frac{\left(2.998 \times 10^{8}\right)^{3}}{6.674 \times 10^{-11}}\left(\frac{5}{96} \pi^{-8 / 3} \times 34.72222^{-11 / 3} \times 103.70375\right)^{3 / 5} \frac{M_{\odot}}{1.989 \times 10^{30}}=36.4917 M_{\odot} \\
& \mathscr{M}_{4}^{-}=\frac{\left(2.998 \times 10^{8}\right)^{3}}{6.674 \times 10^{-11}}\left(\frac{5}{96} \pi^{-8 / 3} \times 37.87879^{-11 / 3} \times 177.24775\right)^{3 / 5} \frac{M_{\odot}}{1.989 \times 10^{30}}=41.5652 M_{\odot} \\
& \mathscr{M}_{5}^{-}=\frac{\left(2.998 \times 10^{8}\right)^{3}}{6.674 \times 10^{-11}}\left(\frac{5}{96} \pi^{-8 / 3} \times 43.47826^{-11 / 3} \times 301.70102\right)^{3 / 5} \frac{M_{\odot}}{1.989 \times 10^{30}}=42.2280 M_{\odot} \\
& \mathscr{M}_{6}^{-}=\frac{\left(2.998 \times 10^{8}\right)^{3}}{6.674 \times 10^{-11}}\left(\frac{5}{96} \pi^{-8 / 3} \times 50.76142^{-11 / 3} \times 584.25788\right)^{3 / 5} \frac{M_{\odot}}{1.989 \times 10^{30}}=44.6521 M_{\odot} \\
& \mathscr{M}_{7}^{-}=\frac{\left(2.998 \times 10^{8}\right)^{3}}{6.674 \times 10^{-11}}\left(\frac{5}{96} \pi^{-8 / 3} \times 64.10256^{-11 / 3} \times 1704.08712\right)^{3 / 5} \frac{M_{\odot}}{1.989 \times 10^{30}}=50.7952 M_{\odot} \\
& \mathscr{M}_{8}^{-}=\frac{\left(2.998 \times 10^{8}\right)^{3}}{6.674 \times 10^{-11}}\left(\frac{5}{96} \pi^{-8 / 3} \times 95.23810^{-11 / 3} \times 5452.51100\right)^{3 / 5} \frac{M_{\odot}}{1.989 \times 10^{30}}=42.7197 M_{\odot} \\
& \mathscr{M}_{9}^{-}=\frac{\left(2.998 \times 10^{8}\right)^{3}}{6.674 \times 10^{-11}}\left(\frac{5}{96} \pi^{-8 / 3} \times 156.25000^{-11 / 3} \times 10588.09569\right)^{3 / 5} \frac{M_{\odot}}{1.989 \times 10^{30}}=21.4062 M_{\odot} \\
& \mathscr{M}_{10}^{-}=\frac{\left(2.998 \times 10^{8}\right)^{3}}{6.674 \times 10^{-11}}\left(\frac{5}{96} \pi^{-8 / 3} \times 212.76600^{-11 / 3} \times 10775.86207\right)^{3 / 5} \frac{M_{\odot}}{1.989 \times 10^{30}}=10.9683 M_{\odot}
\end{aligned}
$$

The estimate of the chirp mass corresponding to the numerical relativistic waveform varies non-monotonically with frequency and cannot be approximately equal, indicating that the frequency distribution of the numerical relativistic waveform does not satisfy the general relativistic Blanche equation. Although the concept of numerical relativistic wave has been beautified and rendered, it has failed to achieve the expected goal.

LIGO announced the detection of gravitational waves in several spiral binary black holes and one spiral binary neutron star system, but avoided the calculation of the distribution and variation law of frequency, which are the most basic physical quantity. The drawing procedure of numerical relativistic gravitational waveform is also lack of the necessary introduction. All conclusions are only qualitative inferences. In fact, only after calculating the frequency distribution and frequency change rate of the signal wave, can we find out the law of frequency distribution and change, so as to determine whether the detected signal wave really tests the general relativity theory.

\section{Classical estimation method for total mass of spiral binaries}

It is possible for different wave sources to produce signal waves with similar frequency variation, and there is no one-to-one correspondence between signal waves and wave sources. The GW150914 signal wave is regarded as the gravitational wave of the merging spiral binary black holes, which is just one of the countless possibilities. Even if it is considered that it is the only possibility, any one of values of the so-called chirps mass of the binary black 
holes has an infinite set of double star masses. The estimation of chirp quality can not accurately obtain the information of a wave source, especially when there are obvious contradictions in the estimation results. Here we introduce the classical estimation results of the total mass of the source when GW150914 signal wave is regarded as the gravitational wave of a spiral double black hole.

As we all know, the calculation results of the classical mechanics of the planetary revolution cycle are consistent with the astronomical observations. A precise gravitational theory is bound to apply both to the strong gravitational field and the weak gravitational field. As an approximate analysis, the estimation of the total mass of a spiral binary black hole by the classical equation of the orbit period will not be too far from the predictions of any exact theory, otherwise there would be an exact dividing point that does not actually exist between the strong gravitational field and the weak gravitational field. If the binary black holes of mass $m_{1}$ and $m_{2}$ are combined into one large black hole, it can be assumed that a particle of mass $m_{0}$ performs a uniform circular motion on the Schwarzschild horizon ${ }^{[23]}$ of the large black hole. The orbital frequency $f_{s}$ of the particle is the Schwarzschild frequency. According to the gravitation Laws and circular motion laws are obtained,

$$
\frac{G\left(m_{1}+m_{2}\right) m_{0}}{R_{s}^{2}}=m_{0} R_{s}\left(2 \pi f_{s}\right)^{2}
$$

Among them, the Schwarzschild radius of a large black hole generated by a binary black hole merger is $R_{s}=$ $2 G\left(m_{1}+m_{2}\right) / c^{2}$, which is replaced by the above formula to get

$$
m_{1}+m_{2}=\frac{c^{3}}{2^{5 / 2} \pi G f_{s}}
$$

The gravitational radiation generated by the microscopic particles around the dense star is very small and has no observation effect. Therefore, it is usually assumed that two spiral black holes merge to generate strong gravitational radiation. The Schwarzschild radii of the two black holes before the merger are $R_{s 1}=2 G m_{1} / c^{2}$ and $R_{s 2}=2 G m_{2} / c^{2}$, respectively. The two black holes run around their mass centers, and detoured Schwarzschild frequency $f_{s}$ still satisfies equation (7).

Assuming that the maximum frequency of the GW150914 signal wave is the Schwarzschild frequency $f_{s}$ of the wave source, then substituting the maximum frequency $f_{s}=230.760 \mathrm{~Hz}$ of the negative strain in Table 1 into equation (7) gives the total mass $m_{1}+m_{2}=9.845 \times$ $10^{31} \mathrm{~kg}=49.497 M_{\odot}$ of two black holes or binary compact stars, where the mass of the sun is $M_{\odot}=1.989 \times 10^{30} \mathrm{~kg}$. And the other estimate of the total mass of the two black holes obtained by substituting the maximum frequency $f_{s}=197.398 \mathrm{~Hz}$ of the positive strain in Table 1 into equation (7) is $59.43 M_{\odot}$. There are some differences between the two estimates. We choose the maximum value that meets the expectations,

$$
m_{1}+m_{2}=59.43 M_{\odot}
$$

The chirp mass of the source estimated by Blanchet frequency equation is very uncertain, so the uncertainty of the total mass is also very large. But the uncertainty of estimating the total mass of the binary black hole according to the Schwarzschild orbit frequency is relatively small. Of course this is just an estimate. Accurate calculation belongs to the content of com quantum theory, which systematically studies the laws of com quantization in nature, what is given therein is the unique value of the statistical average.

\section{Conclusions and comments}

Often an experimental phenomenon can be explained by different theories ${ }^{[24]}$. The important feature of GW150914 signal is the monotonic increase in frequency. We studied in detail the relationship between the frequency distribution of GW150914 signal wave and the generalized relativistic Blanchet frequency equation. It was pointed out that the similarity between GW150914 signal wave and the wave predicted by general relativity is only qualitative. However, frequency distribution and variation law of GW150914 signal do not support the non-linear Blanchet frequency equation, and the difference between them is far beyond the error range. On the other hand, the numerical relativistic waveform deviates too far from the original GW150914 signal waveform. The other LIGO signals do not have the obvious characteristic of monotonous increase in frequency, so they can't be used for accurate spectrum analysis. In short, the LIGO signal does not support the relativistic Blanchet frequency equation of spiral binaries merging gravitational waves.

There is no precise demarcation point between the strong gravitational field and the weak gravitational field. The classical theory of gravitation is based on a large number of astronomical observations. There is no principle difference between the inferences of classical theory describing the gravitational system of black holes and the correct inference beyond classical theory. Therefore, if GW150914 signal wave belongs to gravitational wave of spiral binary stars, the Schwarzschild orbital frequency can be combined with classical theory to estimate the total mass of the wave source. The problem also restores its simple and easy-to-understand nature. The maximum frequency of positive strain of GW150914 signal wave is used to estimate the total mass of the wave source. The result is in line with expectation, but the maximum frequency of negative strain of GW150914 is used to estimate the total mass of the wave source, the result is not in line with expectation. Is there any scientific basis for making a unique choice between the two estimation of the total mass of the wave source? What kind of exact equation does the frequency of the GW150914 signal wave satisfy? How to accurately calculate the gravitational wave source mass and determine the exact position of the gravitational wave source? How to accurately distinguish the different 
gravitational wave signals from the binary black hole, the dense binary star, the multi black hole or the dense multi star gravitational system? What are the necessary and sufficient conditions leading to the formation and merging of spiral binary black holes? All these are urgent problems to be solved by gravitational theory.

Although the frequency distribution of the GW150914 signal wave accords with the motion law of the classical process of spiral binary star, the numerical calculation results of the discrete frequency and rate of change of the positive and negative strain show that only use the quantum number can accurately describe the law of gravitational wave. This is the com quantum theory which is different from the traditional quantum theory. An accurate theory of gravitational waves is bound to be highly consistent with the exact results of experimental observations. The detection of GW150914 signal wave is a scientific fortune, and this achievement will play an important role in the history of gravitational theory for a long time. Based on the monotonic increase of the frequency and strain of the signal wave, it can only be qualitatively judged that the signal wave detected by the laser interferometer gravitational wave detector belongs to the gravitational wave combined by a spiral binary black hole or a helical binary neutron star. The strict proof of the conclusion requires that the numerical analysis results of the observation data with high accuracy conform to the theoretical equation. Recognition of frequency-varying signal wave is a new technology to be developed. The generalized quantization characteristics of GW150914 signal contain a new scientific theory. Perhaps gravitational waves of spiral binaries that will be discovered in the future have the same general quantization law, and the gravitation theory will be developed and perfected.

1 Einstein, A. Die Feldgleichungun der Gravitation. Sitzungsber. K. Preuss. Akad. Wiss., 844-847 (1915).

2 Einstein, A. The foundation of the general theory of relativity. Annalen Phys. 14, 769-822 (1916).

3 Einstein, A. Näherungsweise Integration der Feldgleichungen der Gravitation. Sitzungsber. K. Preuss. Akad. Wiss., 688-696 (1916).

4 Schwarzschild, K. Über das Gravitationsfeld eines Massenpunktes nach der Einsteinschen Theorie. Sitzungsber. K. Preuss. Akad. Wiss., 189-196 (1916).
5 Schwarzschild, K. Über das Gravitationsfeld eines Massenpunktes nach der Einsteinschen Theorie. Sitzungsber. K. Preuss. Akad. Wiss., 424-434 (1916).

6 Einstein, A. Über Gravitationswellen. Sitzungsber. K. Preuss. Akad. Wiss., 154-167 (1918).

7 Kerr, R. P. Gravitational field of a spinning mass as an example of algebraically special metrics. Physical review letters 11, 237-238 (1963).

8 Weber, J. \& Mcvittie, G. C. General Relativity and Gravitational Waves. (Interscience Publishers, Inc, 1961).

9 Abramovici, A. et al. LIGO: The laser interferometer gravitational-wave observatory. Science 256, 325-333 (1992).

10 Harry, G. M. \& Collaboration, L. S. Advanced LIGO: the next generation of gravitational wave detectors. Classical and Quantum Gravity 27, 084006 (2010).

11 Baker, J., Brügmann, B., Campanelli, M., Lousto, C. O. \& Takahashi, R. Plunge waveforms from inspiralling binary black holes. Physical review letters 87, 121103 (2001).

12 Damour, T., Nagar, A., Hannam, M., Husa, S. \& Brügmann, B. Accurate effective-one-body waveforms of inspiralling and coalescing black-hole binaries. Physical Review D 78, 044039 (2008).

13 Hulse, R. \& Taylor, J. A high-sensitivity pulsar survey. The Astrophysical Journal 191, L59 (1974).

14 Blanchet, L., Damour, T., Iyer, B. R., Will, C. M. \& Wiseman, A. G. Gravitational-radiation damping of compact binary systems to second post-Newtonian order. Physical Review Letters 74, 3515 (1995).

15 Abbott, B. P. et al. Observation of gravitational waves from a binary black hole merger. Physical review letters 116, 061102 (2016).

16 Abbott, B. et al. Localization and broadband follow-up of the gravitational-wave transient GW150914. The Astrophysical journal letters 826, L13 (2016).

17 Abbott, B. et al. GW151226: Observation of gravitational waves from a 22-solar-mass binary black hole coalescence. Physical Review Letters 116, 241103 (2016).

18 Scientific, L. et al. GW170104: observation of a 50-solar-mass binary black hole coalescence at redshift 0.2. Physical Review Letters 118, 221101 (2017).

19 Abbott, B. P. et al. GW170814: a three-detector observation of gravitational waves from a binary black hole coalescence. Physical review letters 119, 141101 (2017).

20 Abbott, B. P. et al. GW170817: observation of gravitational waves from a binary neutron star inspiral. Physical Review Letters 119, 161101 (2017).

21 https://losc.ligo.org/events/GW150914/. LIGO Gravitational Wave Strain Data.

22 Sahoo, P. \& Riedel, T. Mean value theorems and functional equations. (World Scientific, 1998).

23 Hawking, S. W. Black holes in general relativity. Communications in Mathematical Physics 25, 152-166 (1972).

24 Wang, R., Zheng, Y. \& Yao, A. Generalized Sagnac effect. Physical Review Letters 93, 143901 (2004).

\section{Appendix A Mathematica Code of FIG3}

$$
\begin{aligned}
& \text { Show ContourPlot }\left[\left\{\pi \dot{f}==\frac{96\left(6.674 \times 10^{-11}\right)^{5 / 3}\left(29 \times 1.989 \times 10^{30}\right)\left(36 \times 1.989 \times 10^{30}\right)(\pi \times f)^{11 / 3}}{5 \times\left(2.998 \times 10^{8}\right)^{5}\left(29 \times 1.989 \times 10^{30}+36 \times 1.989 \times 10^{30}\right)^{1 / 3}}\right.\right. \\
& \times\left\{1-\left(\frac{743}{336}+\frac{11 \times 29 \times 36}{4(29+36)^{2}}\right)\left(\frac{6.674 \times 10^{-11} \pi \times f \times(29+36) \times 1.989 \times 10^{30}}{\left(2.998 \times 10^{8}\right)^{3}}\right)^{2 / 3}+4 \pi \times \frac{6.674 \times 10^{-11} \pi \times f \times(29+36) \times 1.989 \times 10^{30}}{\left(2.998 \times 10^{8}\right)^{3}}\right. \\
& \left.+\left(\frac{34103}{18144}+\frac{13661 \times 29 \times 36}{2016(29+36)^{2}}+\frac{59 \times(29 \times 36)^{2}}{(29+36)^{4}}\right)\left(\frac{6.674 \times 10^{-11} \pi \times f \times(29+36) \times 1.989 \times 10^{30}}{\left(2.998 \times 10^{8}\right)^{3}}\right)^{4 / 3}\right\} \\
& \pi \dot{f}==\frac{96\left(6.674 \times 10^{-11}\right)^{5 / 3}\left(7 \times 1.989 \times 10^{30}\right)\left(58 \times 1.989 \times 10^{30}\right)(\pi \times f)^{11 / 3}}{5 \times\left(2.998 \times 10^{8}\right)^{5}\left(7 \times 1.989 \times 10^{30}+58 \times 1.989 \times 10^{30}\right)^{1 / 3}}
\end{aligned}
$$




$$
\begin{aligned}
& \times\left\{1-\left(\frac{743}{336}+\frac{11 \times 7 \times 58}{4(7+58)^{2}}\right)\left(\frac{6.674 \times 10^{-11} \pi \times f \times(7+58) \times 1.989 \times 10^{30}}{\left(2.998 \times 10^{8}\right)^{3}}\right)^{2 / 3}+4 \pi \times \frac{6.674 \times 10^{-11} \pi \times f \times(7+58) \times 1.989 \times 10^{30}}{\left(2.998 \times 10^{8}\right)^{3}}\right. \\
& \left.+\left(\frac{34103}{18144}+\frac{13661 \times 7 \times 58}{2016(7+58)^{2}}+\frac{59 \times(7 \times 58)^{2}}{(7+58)^{4}}\right)\left(\frac{6.674 \times 10^{-11} \pi \times f \times(7+58) \times 1.989 \times 10^{30}}{\left(2.998 \times 10^{8}\right)^{3}}\right)^{4 / 3}\right\}, \\
& \pi \dot{f}==\frac{96\left(6.674 \times 10^{-11}\right)^{5 / 3}\left(15 \times 1.989 \times 10^{30}\right)\left(50 \times 1.989 \times 10^{30}\right)(\pi \times f)^{11 / 3}}{5 \times\left(2.998 \times 10^{8}\right)^{5}\left(15 \times 1.989 \times 10^{30}+50 \times 1.989 \times 10^{30}\right)^{1 / 3}} \\
& \times\left\{1-\left(\frac{743}{336}+\frac{11 \times 15 \times 50}{4(15+50)^{2}}\right)\left(\frac{6.674 \times 10^{-11} \pi \times f \times(15+50) \times 1.989 \times 10^{30}}{\left(2.998 \times 10^{8}\right)^{3}}\right)^{2 / 3}+4 \pi \times \frac{6.674 \times 10^{-11} \pi \times f \times(15+50) \times 1.989 \times 10^{30}}{\left(2.998 \times 10^{8}\right)^{3}}\right. \\
& \left.+\left(\frac{34103}{18144}+\frac{13661 \times 15 \times 50}{2016(15+50)^{2}}+\frac{59 \times(15 \times 50)^{2}}{(15+50)^{4}}\right)\left(\frac{6.674 \times 10^{-11} \pi \times f \times(15+50) \times 1.989 \times 10^{30}}{\left(2.998 \times 10^{8}\right)^{3}}\right)^{4 / 3}\right\}, \\
& \pi \dot{f}==\frac{96\left(6.674 \times 10^{-11}\right)^{5 / 3}\left(25 \times 1.989 \times 10^{30}\right)\left(40 \times 1.989 \times 10^{30}\right)(\pi \times f)^{11 / 3}}{5 \times\left(2.998 \times 10^{8}\right)^{5}\left(25 \times 1.989 \times 10^{30}+40 \times 1.989 \times 10^{30}\right)^{1 / 3}} \\
& \times\left\{1-\left(\frac{743}{336}+\frac{11 \times 25 \times 40}{4(25+40)^{2}}\right)\left(\frac{6.674 \times 10^{-11} \pi \times f \times(25+40) \times 1.989 \times 10^{30}}{\left(2.998 \times 10^{8}\right)^{3}}\right)^{2 / 3}+4 \pi \times \frac{6.674 \times 10^{-11} \pi \times f \times(25+40) \times 1.989 \times 10^{30}}{\left(2.998 \times 10^{8}\right)^{3}}\right. \\
& \left.+\left(\frac{34103}{18144}+\frac{13661 \times 25 \times 40}{2016(25+40)^{2}}+\frac{59 \times(25 \times 40)^{2}}{(25+40)^{4}}\right)\left(\frac{6.674 \times 10^{-11} \pi \times f \times(25+40) \times 1.989 \times 10^{30}}{\left(2.998 \times 10^{8}\right)^{3}}\right)^{4 / 3}\right\}, \\
& \pi \dot{f}==\frac{96\left(6.674 \times 10^{-11}\right)^{5 / 3}\left(10 \times 1.989 \times 10^{30}\right)\left(55 \times 1.989 \times 10^{30}\right)(\pi \times f)^{11 / 3}}{5 \times\left(2.998 \times 10^{8}\right)^{5}\left(10 \times 1.989 \times 10^{30}+55 \times 1.989 \times 10^{30}\right)^{1 / 3}} \\
& \times\left\{1-\left(\frac{743}{336}+\frac{11 \times 10 \times 55}{4(10+55)^{2}}\right)\left(\frac{6.674 \times 10^{-11} \pi \times f \times(10+55) \times 1.989 \times 10^{30}}{\left(2.998 \times 10^{8}\right)^{3}}\right)^{2 / 3}+4 \pi \times \frac{6.674 \times 10^{-11} \pi \times f \times(10+55) \times 1.989 \times 10^{30}}{\left(2.998 \times 10^{8}\right)^{3}}\right. \\
& \left.+\left(\frac{34103}{18144}+\frac{13661 \times 10 \times 55}{2016(10+55)^{2}}+\frac{59 \times(10 \times 55)^{2}}{(10+55)^{4}}\right)\left(\frac{6.674 \times 10^{-11} \pi \times f \times(10+55) \times 1.989 \times 10^{30}}{\left(2.998 \times 10^{8}\right)^{3}}\right)^{4 / 3}\right\}, \\
& \pi \dot{f}==\frac{96\left(6.674 \times 10^{-11}\right)^{5 / 3}\left(10 \times 1.989 \times 10^{30}\right)\left(65 \times 1.989 \times 10^{30}\right)(\pi \times f)^{11 / 3}}{5 \times\left(2.998 \times 10^{8}\right)^{5}\left(10 \times 1.989 \times 10^{30}+65 \times 1.989 \times 10^{30}\right)^{1 / 3}} \\
& \times\left\{1-\left(\frac{743}{336}+\frac{11 \times 10 \times 65}{4(10+65)^{2}}\right)\left(\frac{6.674 \times 10^{-11} \pi \times f \times(10+65) \times 1.989 \times 10^{30}}{\left(2.998 \times 10^{8}\right)^{3}}\right)^{2 / 3}+4 \pi \times \frac{6.674 \times 10^{-11} \pi \times f \times(10+65) \times 1.989 \times 10^{30}}{\left(2.998 \times 10^{8}\right)^{3}}\right. \\
& \left.+\left(\frac{34103}{18144}+\frac{13661 \times 10 \times 65}{2016(10+65)^{2}}+\frac{59 \times(10 \times 65)^{2}}{(10+65)^{4}}\right)\left(\frac{6.674 \times 10^{-11} \pi \times f \times(10+65) \times 1.989 \times 10^{30}}{\left(2.998 \times 10^{8}\right)^{3}}\right)^{4 / 3}\right\}, \\
& \pi \dot{f}==\frac{96\left(6.674 \times 10^{-11}\right)^{5 / 3}\left(30 \times 1.989 \times 10^{30}\right)\left(50 \times 1.989 \times 10^{30}\right)(\pi \times f)^{11 / 3}}{5 \times\left(2.998 \times 10^{8}\right)^{5}\left(30 \times 1.989 \times 10^{30}+50 \times 1.989 \times 10^{30}\right)^{1 / 3}} \\
& \times\left\{1-\left(\frac{743}{336}+\frac{11 \times 30 \times 50}{4(30+50)^{2}}\right)\left(\frac{6.674 \times 10^{-11} \pi \times f \times(30+50) \times 1.989 \times 10^{30}}{\left(2.998 \times 10^{8}\right)^{3}}\right)^{2 / 3}+4 \pi \times \frac{6.674 \times 10^{-11} \pi \times f \times(30+50) \times 1.989 \times 10^{30}}{\left(2.998 \times 10^{8}\right)^{3}}\right. \\
& \left.\left.+\left(\frac{34103}{18144}+\frac{13661 \times 30 \times 50}{2016(30+50)^{2}}+\frac{59 \times(30 \times 50)^{2}}{(30+50)^{4}}\right)\left(\frac{6.674 \times 10^{-11} \pi \times f \times(30+50) \times 1.989 \times 10^{30}}{\left(2.998 \times 10^{8}\right)^{3}}\right)^{4 / 3}\right\}\right\},
\end{aligned}
$$

$\{f, 0,150\},\{\dot{f}, 0,12000\}$, Axes $\rightarrow$ True, GridLinesStyle $\rightarrow$ Directive[Dashed],

PlotLegends $\rightarrow$ Placed $\left[\left\{\right.\right.$ "BC $\left(m_{1}=29 m_{\odot}, m_{2}=36 m_{\odot}\right) "$, "BC $\left(m_{1}=7 m_{\odot}, m_{2}=58 m_{\odot}\right) "$,

"BC $\left(m_{1}=15 m_{\odot}, m_{2}=50 m_{\odot}\right)$ ", "BC $\left(m_{1}=25 m_{\odot}, m_{2}=40 m_{\odot}\right)$ ", "BC $\left(m_{1}=10 m_{\odot}, m_{2}=55 m_{\odot}\right)$ ",

"BC $\left(m_{1}=10 m_{\odot}, m_{2}=65 m_{\odot}\right)$ ", "BC $\left.\left.\left(m_{1}=30 m_{\odot}, m_{2}=50 m_{\odot}\right) "\right\},\{0.250,0.60\}\right]$, Ticks $\rightarrow\{$ Range $[-10,10,2]\}$,

ContourStyle $\rightarrow\{\{$ Hue[0.7], AbsoluteThickness[3]\}, $\{$ Hue[0.9], AbsoluteThickness[3]\},

$\{$ Hue[0.2], AbsoluteThickness[3]\}, $\{$ Hue[0.3], AbsoluteThickness[3]\},

$\{$ Hue[0.6], AbsoluteThickness[3]\}, \{Hue[0.5], AbsoluteThickness[3]\}, \{Hue[0.8], AbsoluteThickness[3]\}\},

FrameLabel $\rightarrow\left\{\right.$ "f $(\mathrm{Hz})$ ", " $\left.\left.\dot{f}\left(\mathrm{~Hz} \mathrm{~s}^{-1}\right) "\right\}\right]$,

ListLinePlot $[\{\{136.3582345,11831.23042\},\{116.6440307,8657.49422\}$,

$\{92.72359945,3755.061951\},\{79.31794085,2747.763841\},\{68.32265222,1560.827218\}$,

$\{58.44479852,1142.134177\},\{53.04553744,764.1961764\},\{45.37639637,559.1999942\}$,

$\{42.73112738,418.0919129\}\}$, PlotStyle $\rightarrow$ \{Thickness[0.010], RGBColor $[1,0,0]\}$,

PlotLegends $\rightarrow$ Placed[ ["LC of GW150914" $\},\{0.250,0.60\}]]$, AspectRatio $\rightarrow 0.86]$

\section{Appendix B Mathematica Code of FIG4}

ContourPlot $\left[\left\{\pi \times 559.1999942==\frac{96\left(6.674 \times 10^{-11}\right)^{5 / 3}\left(m_{1} \times 1.989 \times 10^{30}\right)\left(m_{2} \times 1.989 \times 10^{30}\right)(\pi \times 45.37639637)^{11 / 3}}{5 \times\left(2.998 \times 10^{8}\right)^{5}\left(m_{1} \times 1.989 \times 10^{30}+m_{2} \times 1.989 \times 10^{30}\right)^{1 / 3}}\right.\right.$

$\times\left\{1-\left(\frac{743}{336}+\frac{11 \times m_{1} \times m_{2}}{4\left(m_{1}+m_{2}\right)^{2}}\right)\left(\frac{6.674 \times 10^{-11} \pi \times 45.37639637\left(m_{1}+m_{2}\right) \times 1.989 \times 10^{30}}{\left(2.998 \times 10^{8}\right)^{3}}\right)^{2 / 3}\right.$ 
$+4 \pi \times \frac{6.674 \times 10^{-11} \pi \times 45.37639637\left(m_{1}+m_{2}\right) \times 1.989 \times 10^{30}}{\left(2.998 \times 10^{8}\right)^{3}}$

$\left.+\left(\frac{34103}{18144}+\frac{13661 \times m_{1} \times m_{2}}{2016\left(m_{1}+m_{2}\right)^{2}}+\frac{59 \times\left(m_{1} \times m_{2}\right)^{2}}{\left(m_{1}+m_{2}\right)^{4}}\right)\left(\frac{6.674 \times 10^{-11} \pi \times 45.37639637\left(m_{1}+m_{2}\right) \times 1.989 \times 10^{30}}{\left(2.998 \times 10^{8}\right)^{3}}\right) 4 / 3\right\}$,

$\pi \times 1142.134177==\frac{96\left(6.674 \times 10^{-11}\right)^{5 / 3}\left(m_{1} \times 1.989 \times 10^{30}\right)\left(m_{2} \times 1.989 \times 10^{30}\right)(\pi \times 58.44479852)^{11 / 3}}{5 \times\left(2.998 \times 10^{8}\right)^{5}\left(m_{1} \times 1.989 \times 10^{30}+m_{2} \times 1.989 \times 10^{30}\right)^{1 / 3}}$

$\times\left\{1-\left(\frac{743}{336}+\frac{11 \times m_{1} \times m_{2}}{4\left(m_{1}+m_{2}\right)^{2}}\right)\left(\frac{6.674 \times 10^{-11} \pi \times 58.44479852\left(m_{1}+m_{2}\right) \times 1.989 \times 10^{30}}{\left(2.998 \times 10^{8}\right)^{3}}\right)^{2 / 3}\right.$

$+4 \pi \times \frac{6.674 \times 10^{-11} \pi \times 58.44479852\left(m_{1}+m_{2}\right) \times 1.989 \times 10^{30}}{\left(2.998 \times 10^{8}\right)^{3}}$

$\left.+\left(\frac{34103}{18144}+\frac{13661 \times m_{1} \times m_{2}}{2016\left(m_{1}+m_{2}\right)^{2}}+\frac{59 \times\left(m_{1} \times m_{2}\right)^{2}}{\left(m_{1}+m_{2}\right)^{4}}\right)\left(\frac{6.674 \times 10^{-11} \pi \times 58.44479852\left(m_{1}+m_{2}\right) \times 1.989 \times 10^{30}}{\left(2.998 \times 10^{8}\right)^{3}}\right) 4 / 3\right\}$, $\pi \times 2747.763841==\frac{96\left(6.674 \times 10^{-11}\right)^{5 / 3}\left(m_{1} \times 1.989 \times 10^{30}\right)\left(m_{2} \times 1.989 \times 10^{30}\right)(\pi \times 79.31794085)^{11 / 3}}{5 \times\left(2.998 \times 10^{8}\right)^{5}\left(m_{1} \times 1.989 \times 10^{30}+m_{2} \times 1.989 \times 10^{30}\right)^{1 / 3}}$

$\times\left\{1-\left(\frac{743}{336}+\frac{11 \times m_{1} \times m_{2}}{4\left(m_{1}+m_{2}\right)^{2}}\right)\left(\frac{6.674 \times 10^{-11} \pi \times 79.31794085\left(m_{1}+m_{2}\right) \times 1.989 \times 10^{30}}{\left(2.998 \times 10^{8}\right)^{3}}\right) 2 / 3\right.$

$+4 \pi \times \frac{6.674 \times 10^{-11} \pi \times 79.31794085\left(m_{1}+m_{2}\right) \times 1.989 \times 10^{30}}{\left(2.998 \times 10^{8}\right)^{3}}$

$\left.+\left(\frac{34103}{18144}+\frac{13661 \times m_{1} \times m_{2}}{2016\left(m_{1}+m_{2}\right)^{2}}+\frac{59 \times\left(m_{1} \times m_{2}\right)^{2}}{\left(m_{1}+m_{2}\right)^{4}}\right)\left(\frac{6.674 \times 10^{-11} \pi \times 79.31794085\left(m_{1}+m_{2}\right) \times 1.989 \times 10^{30}}{\left(2.998 \times 10^{8}\right)^{3}}\right) 4 / 3\right\}$, $\pi \times 8657.49422==\frac{96\left(6.674 \times 10^{-11}\right)^{5 / 3}\left(m_{1} \times 1.989 \times 10^{30}\right)\left(m_{2} \times 1.989 \times 10^{30}\right)(\pi \times 116.6440307)^{11 / 3}}{5 \times\left(2.998 \times 10^{8}\right)^{5}\left(m_{1} \times 1.989 \times 10^{30}+m_{2} \times 1.989 \times 10^{30}\right)^{1 / 3}}$

$\times\left\{1-\left(\frac{743}{336}+\frac{11 \times m_{1} \times m_{2}}{4\left(m_{1}+m_{2}\right)^{2}}\right)\left(\frac{6.674 \times 10^{-11} \pi \times 116.6440307\left(m_{1}+m_{2}\right) \times 1.989 \times 10^{30}}{\left(2.998 \times 10^{8}\right)^{3}}\right)^{2 / 3}\right.$

$+4 \pi \times \frac{6.674 \times 10^{-11} \pi \times 116.6440307\left(m_{1}+m_{2}\right) \times 1.989 \times 10^{30}}{\left(2.998 \times 10^{8}\right)^{3}}$

$\left.+\left(\frac{34103}{18144}+\frac{13661 \times m_{1} \times m_{2}}{2016\left(m_{1}+m_{2}\right)^{2}}+\frac{59 \times\left(m_{1} \times m_{2}\right)^{2}}{\left(m_{1}+m_{2}\right)^{4}}\right)\left(\frac{6.674 \times 10^{-11} \pi \times 116.6440307\left(m_{1}+m_{2}\right) \times 1.989 \times 10^{30}}{\left(2.998 \times 10^{8}\right)^{3}}\right) 4 / 3\right\}$,

$\pi \times 418.0919129==\frac{96\left(6.674 \times 10^{-11}\right)^{5 / 3}\left(m_{1} \times 1.989 \times 10^{30}\right)\left(m_{2} \times 1.989 \times 10^{30}\right)(\pi \times 42.73112738)^{11 / 3}}{5 \times\left(2.998 \times 10^{8}\right)^{5}\left(m_{1} \times 1.989 \times 10^{30}+m_{2} \times 1.989 \times 10^{30}\right)^{1 / 3}}$

$\times\left\{1-\left(\frac{743}{336}+\frac{11 \times m_{1} \times m_{2}}{4\left(m_{1}+m_{2}\right)^{2}}\right)\left(\frac{6.674 \times 10^{-11} \pi \times 42.73112738\left(m_{1}+m_{2}\right) \times 1.989 \times 10^{30}}{\left(2.998 \times 10^{8}\right)^{3}}\right) 2 / 3\right.$

$+4 \pi \times \frac{6.674 \times 10^{-11} \pi \times 42.73112738\left(m_{1}+m_{2}\right) \times 1.989 \times 10^{30}}{\left(2.998 \times 10^{8}\right)^{3}}$

$\left.+\left(\frac{34103}{18144}+\frac{13661 \times m_{1} \times m_{2}}{2016\left(m_{1}+m_{2}\right)^{2}}+\frac{59 \times\left(m_{1} \times m_{2}\right)^{2}}{\left(m_{1}+m_{2}\right)^{4}}\right)\left(\frac{6.674 \times 10^{-11} \pi \times 42.73112738\left(m_{1}+m_{2}\right) \times 1.989 \times 10^{30}}{\left(2.998 \times 10^{8}\right)^{3}}\right) 4 / 3\right\}$,

$\pi \times 764.1961764==\frac{96\left(6.674 \times 10^{-11}\right)^{5 / 3}\left(m_{1} \times 1.989 \times 10^{30}\right)\left(m_{2} \times 1.989 \times 10^{30}\right)(\pi \times 53.04553744)^{11 / 3}}{5 \times\left(2.998 \times 10^{8}\right)^{5}\left(m_{1} \times 1.989 \times 10^{30}+m_{2} \times 1.989 \times 10^{30}\right)^{1 / 3}}$

$\times\left\{1-\left(\frac{743}{336}+\frac{11 \times m_{1} \times m_{2}}{4\left(m_{1}+m_{2}\right)^{2}}\right)\left(\frac{6.674 \times 10^{-11} \pi \times 53.04553744\left(m_{1}+m_{2}\right) \times 1.989 \times 10^{30}}{\left(2.998 \times 10^{8}\right)^{3}}\right) 2 / 3\right.$

$+4 \pi \times \frac{6.674 \times 10^{-11} \pi \times 53.04553744\left(m_{1}+m_{2}\right) \times 1.989 \times 10^{30}}{\left(2.998 \times 10^{8}\right)^{3}}$

$\left.+\left(\frac{34103}{18144}+\frac{13661 \times m_{1} \times m_{2}}{2016\left(m_{1}+m_{2}\right)^{2}}+\frac{59 \times\left(m_{1} \times m_{2}\right)^{2}}{\left(m_{1}+m_{2}\right)^{4}}\right)\left(\frac{6.674 \times 10^{-11} \pi \times 53.04553744\left(m_{1}+m_{2}\right) \times 1.989 \times 10^{30}}{\left(2.998 \times 10^{8}\right)^{3}}\right) 4 / 3\right\}$, $\pi \times 1560.827218==\frac{96\left(6.674 \times 10^{-11}\right)^{5 / 3}\left(m_{1} \times 1.989 \times 10^{30}\right)\left(m_{2} \times 1.989 \times 10^{30}\right)(\pi \times 68.32265222)^{11 / 3}}{5 \times\left(2.998 \times 10^{8}\right)^{5}\left(m_{1} \times 1.989 \times 10^{30}+m_{2} \times 1.989 \times 10^{30}\right)^{1 / 3}}$

$\times\left\{1-\left(\frac{743}{336}+\frac{11 \times m_{1} \times m_{2}}{4\left(m_{1}+m_{2}\right)^{2}}\right)\left(\frac{6.674 \times 10^{-11} \pi \times 68.32265222\left(m_{1}+m_{2}\right) \times 1.989 \times 10^{30}}{\left(2.998 \times 10^{8}\right)^{3}}\right)^{2 / 3}\right.$

$+4 \pi \times \frac{6.674 \times 10^{-11} \pi \times 68.32265222\left(m_{1}+m_{2}\right) \times 1.989 \times 10^{30}}{\left(2.998 \times 10^{8}\right)^{3}}$

$\left.+\left(\frac{34103}{18144}+\frac{13661 \times m_{1} \times m_{2}}{2016\left(m_{1}+m_{2}\right)^{2}}+\frac{59 \times\left(m_{1} \times m_{2}\right)^{2}}{\left(m_{1}+m_{2}\right)^{4}}\right)\left(\frac{6.674 \times 10^{-11} \pi \times 68.32265222\left(m_{1}+m_{2}\right) \times 1.989 \times 10^{30}}{\left(2.998 \times 10^{8}\right)^{3}}\right) 4 / 3\right\}$, $\pi \times 3755.061951==\frac{96\left(6.674 \times 10^{-11}\right)^{5 / 3}\left(m_{1} \times 1.989 \times 10^{30}\right)\left(m_{2} \times 1.989 \times 10^{30}\right)(\pi \times 92.72359945)^{11 / 3}}{5 \times\left(2.998 \times 10^{8}\right)^{5}\left(m_{1} \times 1.989 \times 10^{30}+m_{2} \times 1.989 \times 10^{30}\right)^{1 / 3}}$

$\times\left\{1-\left(\frac{743}{336}+\frac{11 \times m_{1} \times m_{2}}{4\left(m_{1}+m_{2}\right)^{2}}\right)\left(\frac{6.674 \times 10^{-11} \pi \times 92.72359945\left(m_{1}+m_{2}\right) \times 1.989 \times 10^{30}}{\left(2.998 \times 10^{8}\right)^{3}}\right)^{2 / 3}\right.$

$+4 \pi \times \frac{6.674 \times 10^{-11} \pi \times 92.72359945\left(m_{1}+m_{2}\right) \times 1.989 \times 10^{30}}{\left(2.998 \times 10^{8}\right)^{3}}$

$\left.+\left(\frac{34103}{18144}+\frac{13661 \times m_{1} \times m_{2}}{2016\left(m_{1}+m_{2}\right)^{2}}+\frac{59 \times\left(m_{1} \times m_{2}\right)^{2}}{\left(m_{1}+m_{2}\right)^{4}}\right)\left(\frac{6.674 \times 10^{-11} \pi \times 92.72359945\left(m_{1}+m_{2}\right) \times 1.989 \times 10^{30}}{\left(2.998 \times 10^{8}\right)^{3}}\right) 4 / 3\right\}$,

$\pi \times 11831.23042==\frac{96\left(6.674 \times 10^{-11}\right)^{5 / 3}\left(m_{1} \times 1.989 \times 10^{30}\right)\left(m_{2} \times 1.989 \times 10^{30}\right)(\pi \times 136.3582345)^{11 / 3}}{5 \times\left(2.998 \times 10^{8}\right)^{5}\left(m_{1} \times 1.989 \times 10^{30}+m_{2} \times 1.989 \times 10^{30}\right)^{1 / 3}}$

$\times\left\{1-\left(\frac{743}{336}+\frac{11 \times m_{1} \times m_{2}}{4\left(m_{1}+m_{2}\right)^{2}}\right)\left(\frac{6.674 \times 10^{-11} \pi \times 136.3582345\left(m_{1}+m_{2}\right) \times 1.989 \times 10^{30}}{\left(2.998 \times 10^{8}\right)^{3}}\right)^{2 / 3}\right.$ 
$+4 \pi \times \frac{6.674 \times 10^{-11} \pi \times 136.3582345\left(m_{1}+m_{2}\right) \times 1.989 \times 10^{30}}{\left(2.998 \times 10^{8}\right)^{3}}$

$\left.\left.+\left(\frac{34103}{18144}+\frac{13661 \times m_{1} \times m_{2}}{2016\left(m_{1}+m_{2}\right)^{2}}+\frac{59 \times\left(m_{1} \times m_{2}\right)^{2}}{\left(m_{1}+m_{2}\right)^{4}}\right)\left(\frac{6.674 \times 10^{-11} \pi \times 136.3582345\left(m_{1}+m_{2}\right) \times 1.989 \times 10^{30}}{\left(2.998 \times 10^{8}\right)^{3}}\right) 4 / 3\right\}\right\}$,

$\left\{m_{1}, 6,130\right\},\left\{m_{2}, 6,130\right\}$, GridLinesStyle $\rightarrow$ Directive[Dashed], Axes $\rightarrow$ False,

GridLinesStyle $\rightarrow$ Directive[Dashed], PlotLegends $\rightarrow$ Placed[\{ "Peak $(45.376 \mathrm{~Hz}, 559.200 \mathrm{~Hz} / \mathrm{s})$ ",

"Peak(58.445Hz,1142.134Hz/s)", "Peak(79.318Hz,2747.764Hz/s)", "Peak(116.644Hz,8657.494Hz/s)",

"Trough(42.731Hz,418.092Hz/s)", "Trough(53.046Hz,764.196Hz/s)", "Trough $\left(68.323 \mathrm{~Hz}, 1560.827 \mathrm{~Hz}^{2}\right)$ ",

"Trough $\left(92.724 \mathrm{~Hz}, 755.062 \mathrm{~Hz}^{2}\right) "$, "Trough $\left.\left.\left(136.358 \mathrm{~Hz}, 11831.2 \mathrm{~Hz}^{2}\right) "\right\},\{0.76,0.60\}\right]$,

Ticks $\rightarrow\{$ Range $[-10,10,2]\}$, ContourStyle $\rightarrow\{\{$ Hue[0.1], AbsoluteThickness[3] $\}$,

$\{$ Hue[0.2], AbsoluteThickness[3]\}, \{Hue[0.3], AbsoluteThickness[3]\},

$\{$ Hue[0.4], AbsoluteThickness[3]\}, \{Hue[0.5], AbsoluteThickness[3]\},

$\{$ Hue[0.60], AbsoluteThickness[3]\}, \{Hue[0.7], AbsoluteThickness[3]\},

$\{$ Hue[0.8], AbsoluteThickness[3]\}, \{Hue[0.9], AbsoluteThickness[3]\}\},

FrameLabel $\rightarrow\left\{" m_{1}\left(m_{\odot}\right) ", " m_{2}\left(m_{\odot}\right) "\right\}$, AspectRatio $\left.\rightarrow 0.86\right]$ 